\title{
A non-LTE study of neutral and singly-ionized calcium in late-type stars ${ }^{\star}$
}

\author{
L. Mashonkina ${ }^{1,2}$, A. J. Korn ${ }^{3}$, and N. Przybilla ${ }^{4}$ \\ ${ }^{1}$ Institut für Astronomie und Astrophysik der Universität München, Scheinerstr. 1, 81679 München, Germany \\ e-mail: lyuda@usm.lmu.de \\ 2 Institute of Astronomy, Russian Academy of Science, Pyatnitskaya 48, 119017 Moscow, Russia \\ e-mail: lima@inasan.ru \\ 3 Department of Astronomy and Space Physics, Uppsala University, Box 515, 75120 Uppsala, Sweden \\ ${ }^{4}$ Dr. Remeis-Sternwarte Bamberg, Sternwartstrasse 7, 96049 Bamberg, Germany
}

Received 10 July 2006 / Accepted 4 September 2006

ABSTRACT

\begin{abstract}
Aims. Non-local thermodynamical equilibrium (NLTE) line formation for neutral and singly-ionized calcium is considered through a range of spectral types when the $\mathrm{Ca}$ abundance varies from the solar value down to $[\mathrm{Ca} / \mathrm{H}]=-5$. We evaluate the influence of departures from LTE on $\mathrm{Ca}$ abundance determinations and inspect the possibility of using $\mathrm{Ca}$ I / Ca II line-strength ratios as indicators of surface gravity for extremely metal-poor stars.

Methods. A comprehensive model atom for $\mathrm{Ca}$ I and $\mathrm{Ca}$ II is presented. Accurate radiative and electron collisional atomic data are incorporated. The role of inelastic collisions with hydrogen atoms in the statistical equilibrium of $\mathrm{Ca} / \mathrm{II}$ is estimated empirically from inspection of their different influences on the Ca I and Ca II lines in selected stars with well determined stellar parameters and high-quality observed spectra.

Results. The dependence of NLTE effects on the atmospheric parameters is discussed. Departures from LTE significantly affect the profiles of $\mathrm{Ca}$ I lines over the whole range of stellar parameters being considered. However, at $[\mathrm{Ca} / \mathrm{H}] \geq-2$, NLTE abundance correction of individual lines have a low absolute value due to the different influence of NLTE effects on line wings and the line core. At lower Ca abundances, NLTE leads to systematically depleted total absorption in the line and positive abundance corrections, exceeding +0.5 dex for $\mathrm{Ca}$ I $\lambda 4226$ at $[\mathrm{Ca} / \mathrm{H}]=-4.9$. In contrast, the NLTE effects strengthen the Ca II lines and lead to negative abundance corrections. NLTE corrections are small, $\leq 0.02 \mathrm{dex}$, for the Ca II resonance lines, and they grow in absolute value with decreasing $\mathrm{Ca}$ abundance for the IR lines of multiplet $3 \mathrm{~d}-4 \mathrm{p}$, exceeding 0.4 dex in the metal-poor models with $[\mathrm{Fe} / \mathrm{H}] \leq-3$. As a test and first application of the Ca I/II model atom, Ca abundances are determined on the basis of plane-parallel LTE model atmospheres for the Sun, Procyon (F IV-V), and seven metal-poor stars, using high S/N and high-resolution spectra at visual and near-IR wavelengths. Lines of $\mathrm{Ca}$ I and Ca II give consistent abundances for all objects (except Procyon) when collisions with hydrogen atoms are taken into account. The derived absolute solar $\mathrm{Ca}$ abundance (from Ca I and $\mathrm{Ca}$ II lines) is $\log \varepsilon_{\mathrm{Ca}, \odot}=6.38 \pm 0.06$. For Procyon, the mean $\mathrm{Ca}$ abundance from $\mathrm{Ca}$ I lines is markedly subsolar, $[\mathrm{Ca} / \mathrm{H}]=-0.14 \pm 0.03$. All metal-poor stars within our sample show an overabundance of calcium relative to iron with $[\mathrm{Ca} / \mathrm{Fe}]$ abundance ratios of 0.26 to 0.46 that are typical of the halo population. The $W(\mathrm{Ca}$ I4226) / $W$ (Ca II8498) equivalent width ratio is predicted to be sensitive to surface gravity for extremely metal-poor stars, while this is not the case for the ratio involving the Ca II resonance line(s).
\end{abstract}

Key words. line: formation - Sun: atmosphere - stars: abundances - stars: late-type - line: profiles

\section{Introduction}

Calcium is one of the best observable chemical elements in latetype stars. The subordinate lines of neutral Ca located in the relatively uncrowded yellow-to-red spectral regions are suitable for spectroscopic analysis over a wide range of $\mathrm{Ca}$ abundance from super-solar values down to $[\mathrm{Ca} / \mathrm{H}]=-4$ (Cayrel et al. 2004). The resonance lines of CaI at $\lambda 4226 \AA$ and Ca II at $\lambda 3933$ and $3968 \AA$ lie in the visual spectral range and can be measured even in extremely metal-poor stars with metallicity $[\mathrm{Fe} / \mathrm{H}]<$ -5 (Christlieb et al. 2002; Frebel et al. 2005). In such stars, $\mathrm{Ca}$ is the only chemical element that is visible in two ionization stages, and the $\mathrm{Ca}$ I and $\mathrm{Ca}$ II lines can be potent tools in

* Based on observations collected at the European Southern Observatory, Chile, 67.D-0086A, and the German Spanish Astronomical Center, Calar Alto, Spain. deriving accurate values for fundamental stellar parameters and for the $\mathrm{Ca}$ abundance itself. Calcium is an important chemical element for studing the history of $\alpha$-process nucleosynthesis in the Galaxy.

The subordinate lines of ionized $\mathrm{Ca}$ at $\lambda 8498,8542$, and $8662 \AA$ are among the strongest features in the near-infrared spectra of late-type stars with metallicity down to $[\mathrm{Fe} / \mathrm{H}]=$ -3 (Mallik 1997). The CaII triplet lies at the focus of cool star research with the advent of large spectroscopic surveys of the Galaxy like the Radial Velocity Experiment (RAVE, Steinmetz et al. 2006) and the upcoming ESA Gaia satellite mission (Perryman et al. 2001). These broad lines are also powerful abundance and metallicity indicators out to large distances via medium-resolution spectroscopy, favorably located near the flux maximum of red giants. Applications range from quantitative analyses of individual stars in globular clusters (e.g. 
Armandroff \& da Costa 1991; Rutledge et al. 1997) to stars and stellar populations in nearby galaxies (e.g. Tolstoy et al. 2001; Ibata et al. 2005). Moreover, the Ca II triplet is useful for analyzing of unresolved stellar systems like early-type galaxies (e.g. Saglia et al. 2002). However, as an increasing number of highquality (echelle) spectra of stars and unresolved stellar systems becomes available, an equally high level in the theoretical modelling is required.

Previous investigations of the statistical equilibrium (SE) of neutral $\mathrm{Ca}$ in the Sun and Procyon (Watanabe \& Steenbock 1985) and in the models with effective temperatures $T_{\text {eff }}=$ $4500 \mathrm{~K}-6200 \mathrm{~K}$, surface gravities $\log g=4.5-1.0$ and metal abundance, $[\mathrm{Fe} / \mathrm{H}]=0$ and -1 (Drake 1991) found rather small departures from LTE. Drake (1991) concluded that "overionization effects (for $\mathrm{Ca}$ I) are not expected to increase in severity in metal-poor stars". Non-local thermodynamical equilibrium (NLTE) analysis of neutral Ca in the sample of 252 dwarf and subgiant stars was performed by Idiart \& Thevenin (2000). Previous NLTE investigations for Ca II were only concerned with the resonance lines in the Sun (we cite only the first paper, Shine \& Linsky 1974) and the IR lines of multiplet 3d-4p ( $\lambda$ 8498, 8542 and $8662 \AA$ ) in moderately metal-poor stars by Jørgensen et al. (1992) for $[\mathrm{Fe} / \mathrm{H}] \geq-1$ and by Andretta et al. (2005) for $[\mathrm{Fe} / \mathrm{H}] \geq-2$.

The need for a new analysis is motivated by two points. First, a fairly extensive set of accurate atomic data on photoionization cross-sections and oscillator strengths was recently calculated in the Opacity Project (OP; see Seaton et al. 1994 for a general review). We include 66 terms of $\mathrm{Ca} \mathrm{I}$ in the model atom, in contrast to 16 terms in the works of Watanabe \& Steenbock (1985) and Drake (1991), and 36 terms of Ca II in contrast to 3 terms considered by Jørgensen et al. (1992) and Andretta et al. (2005). This allows us to investigate the detailed line formation of the high excitation lines of Ca I and Ca II. All our results are based on line-profile analysis, and they take advantage of the advanced theory of collisional broadening by atomic hydrogen treated recently by Anstee \& O'Mara (1995), Barklem \& O'Mara (1997, 1998), and Barklem et al. (1998). Hereafter, these four important papers are referred to collectively as $A \& O^{\prime} M$. The second point is connected with an extension of the metallicity range observed in stars down to $[\mathrm{Fe} / \mathrm{H}]=-5.45$ (Aoki et al. 2006). It is highly desirable to have an understanding of the likely influence of any departures from LTE over this whole range. In stars differing significantly in their metal abundance, different subsets of $\mathrm{Ca}$ lines are used to calculate the $\mathrm{Ca}$ II/Ca I ionization equilibrium and $\mathrm{Ca}$ abundance. We investigate here the NLTE line formation of our extended list of $\mathrm{Ca}$ I and $\mathrm{Ca}$ II lines in the metallicity range between $[\mathrm{Fe} / \mathrm{H}]=0$ and -4.34 . An exception is the resonance lines of $\mathrm{Ca}$ I and $\mathrm{Ca}$ II and the Ca II lines of multiplet $3 \mathrm{~d}-4 \mathrm{p}$ in solar metallicity stars: their cores and inner wings are most probably influenced by the chromospheric temperature rise and a non-thermal and depth-dependent chromospheric velocity field neither of which are part of the homogeneous photospheric models used in this study.

For the statistical equilibrium of atoms in cool stars, an important issue debated for decades, from Gehren (1975) to Belyaev \& Barklem (2003), is the role of inelastic collisions with hydrogen atoms. One of our aims is to empirically constrain the efficiency of this type of collision in the SE of CaI/II from inspection of their different influences on the $\mathrm{Ca}$ I and $\mathrm{Ca}$ II lines in the Sun, Procyon, and seven metal-poor $(-1.35 \geq[\mathrm{Fe} / \mathrm{H}] \geq$ -2.43) stars with well-determined stellar parameters and highquality observed spectra.
The paper is organized as follows. The model atom of $\mathrm{Ca}$ I/II and atomic data are described in Sect. 2. Departures from LTE for CaI and Ca II, NLTE trends with metallicity, effective temperature and surface gravity, and errors in the NLTE results caused by the uncertainties of atomic data are discussed in Sect. 3. In Sect. 4, the solar $\mathrm{Ca}$ spectrum is studied to provide the basis for further differential analysis of stellar spectra. We determine the absolute solar $\mathrm{Ca}$ abundance using four different sets of oscillator strengths based on laboratory measurements, OP calculations, and collected from the NIST (http://physics.nist.gov/PhysRefData) and VALD (Kupka et al. 1999) databases and, thus, estimate the accuracy of the available atomic data. Observations and stellar parameters of our sample of stars are described in Sect. 5. For the selected stars, Sect. 6 investigates whether or not $\mathrm{Ca}$ abundances derived from the $\mathrm{Ca}$ I and Ca II lines agree. NLTE formation of the $\mathrm{Ca}$ I resonance line in the atmospheres of metal-poor stars is tested in Sect. 7. The predictions are made in Sect. 8 for the $\mathrm{Ca}$ I/Ca II line-strength ratios as indicators of surface gravity for extremely metal-poor stars. Our recommendations and conclusions are given in Sect. 9.

\section{The method of NLTE calculations for Ca I/II}

In this section, we treat the model atom of $\mathrm{Ca} / \mathrm{II}$ and briefly describe the programs used for computing the CaI/II level populations and spectral line profiles.

\subsection{The model atom}

Energy levels. Calcium is almost completely ionized throughout the atmosphere of stars with an effective temperature between $5000 \mathrm{~K}$ and $6500 \mathrm{~K}$, and a fraction of $\mathrm{Ca}$ I does not exceed several parts in a thousand. Minority species are particularly sensitive to NLTE effects because any small change in the ionization rates changes their populations a lot. In order to provide close collisional coupling of $\mathrm{Ca}$ I to the continuum electron reservoir and consequently establish a realistic ionization balance between the atomic and singly-ionized species, the atomic model for calcium has to be fairly complete. Energy levels up to $0.17 / 0.67 \mathrm{eV}$ below the ionization threshold are explicitly included in our $\mathrm{Ca}$ I/II model atom. Only the ground state of Ca III is considered. The Ca I levels belong to singlet and triplet terms of the $4 \mathrm{~s} n l$ ( $n \leq 9$ and $l \leq 4), 3 \mathrm{~d} n l(n l=4 \mathrm{p}$ and $3 \mathrm{~d})$, and $4 \mathrm{p}^{2}$ electronic configurations. Triplet fine structure is neglected except for the $4 \mathrm{p}^{3} \mathrm{P}^{\circ}$ and $3 \mathrm{~d}^{3} \mathrm{D}$ splitting. Singlet and triplet $n g, 8 \mathrm{f}, 9 \mathrm{~d}$, and 9f levels are combined into a single level due to their small energy differences. The final model atom includes 63 levels of Ca I. The Ca II levels belong to doublet terms of the $n l(n=4-9$ and $l \leq 5), 10 \mathrm{~s}$, and $10 \mathrm{p}$ electronic configurations. Fine structure splitting sub-levels are included explicitly for the terms $3 \mathrm{~d}^{2} \mathrm{D}$, $4 \mathrm{p}^{2} \mathrm{P}^{\circ}$, and $4 \mathrm{~d}^{2} \mathrm{D}$. The $n g$ and $n h(n=7,8$, and 9$)$ levels are combined into a single level. The final model atom contains 37 levels of Ca II.

The energy levels were taken from the NIST atomic spectra database (Sugar \& Corliss 1985) for all the Ca I electronic configurations with $n \leq 5$ and Ca II electronic configurations with $n \leq 6$. For the remainder, Opacity-Project data from the TOPBASE database (Cunto \& Mendoza 1992) are used. The corresponding term diagram is shown in Fig. 1.

Radiative transitions. Our NLTE calculations rely heavily on the OP radiative data. They cover the whole range of allowed transitions in our model atom. Currently available OP calculations do not include the effects of spin-orbit interaction, and 

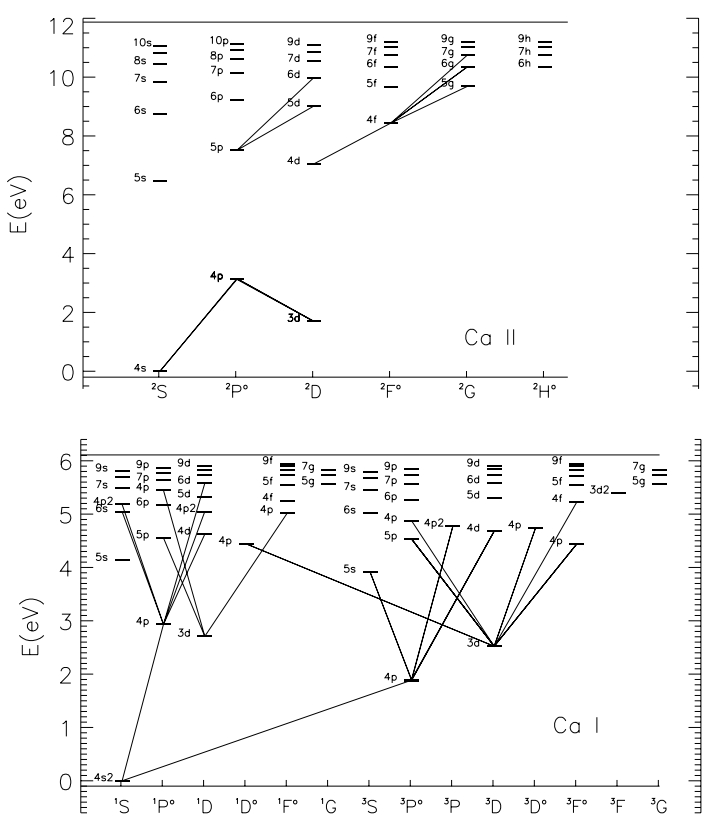

Fig. 1. The Ca I/II model atom. The Ca I and Ca II spectral lines used in $\mathrm{Ca}$ abundance analysis arise in the transitions shown as continuous lines.

only average-multiplet oscillator strengths are reported. Where required, we decompose the $L S$ multiplet averages into their $L S J$ fine structure components in correspondence with their relative line strengths. Additional radiative coupling between the different spin systems of $\mathrm{Ca} \mathrm{I}$ is provided by the transition $4 s^{2}-4 p^{3} \mathrm{P}_{1}^{\circ}$ (Drozdowski et al. 1997) and by all intercombination transitions listed by Smith \& Raggett (1981). The radiative transfer equation is solved, in total, for 421 line transitions in $\mathrm{Ca}$ I and 213 transitions in Ca II.

We compared OP $f_{i j}$ for CaI with the experimental values from Shabanova (1963), Smith \& Gallagher (1966), Smith \& O’Neil (1975), Smith \& Raggett (1981), and Smith (1988) for a total of 24 multiplets with $\log g f$ ranging between 0.73 and -2.25 . The difference $\Delta \log g f$ (laboratory - OP) shows no correlation with the multiplet strength, and the mean value equals $-0.07 \pm 0.28$. For Ca II, measured oscillator strengths are available only for the multiplets $4 s-4 p$ and $3 d-4 p$ (Theodosiou 1989). They agree within $12 \%$ with the corresponding OP values. The OP data for the remaining $\mathrm{Ca}$ I and $\mathrm{Ca}$ II transitions were compared with the calculations of Kurucz (1992). For the 151 strongest $(\log g f(\mathrm{OP})>-2.5)$ Ca I multiplets, the mean difference $\Delta \log g f($ Kurucz - OP $)=-0.03 \pm 0.93$. The Kurucz calculations predict, on average, lower transition probabilities compared to the OP data for the 58 strongest $(\log g f(\mathrm{OP})>-2.5)$ Ca II multiplets, and the mean difference $\Delta \log g f$ (Kurucz $\mathrm{OP})=-0.17 \pm 0.86$. With respect to systematical errors, the OP data for $\mathrm{Ca}$ I and $\mathrm{Ca}$ II transitions are expected to be accurate within $15 \%$. Additional arguments for the validity of this statement come from the analysis of solar Ca spectrum (see Sect. 4).

Photoionization from all energy levels is treated by utilizing OP cross-sections as available through the TOPBASE database. Cross-sections for the combined $n g h$ levels in Ca II are assumed to be equal to that for the corresponding $n g$ levels.

Collisional transitions. In our calculations we take into account inelastic collisions with electrons and hydrogen atoms leading to both excitation and ionization. Drawin's (1968) formula as described by Steenbock \& Holweger (1984) is widely used to calculate hydrogenic collisions, and it suggests that their influence is comparable to electron impact. Recently it was shown both experimentally (Belyaev et al. 1999, for Na I) and theoretically (Belyaev \& Barklem 2003, for Li I) that Drawin's formula overestimates the collision rate of the resonance transitions by several orders of magnitude. However, for SE calculations, estimates are required for transitions between all states that might affect the population of the states of interest. In this study, we constrain the efficiency of hydrogenic collisions empirically. It is represented by a scaling factor $S_{\mathrm{H}}$ applied to Steenbock $\&$ Holweger's formula. The cross-sections calculated using this formula were multiplied by $S_{\mathrm{H}}=0$ (no hydrogenic collisions), $0.01,0.1$, and 1 in order to make the $\mathrm{Ca}$ abundances derived from the Ca I and Ca II lines in the Sun and selected stars consistent.

For electron impact excitation, detailed results are available from the $R$-matrix calculations of Samson \& Berrington (2001) for the transitions from the ground state to all the excited levels up to $3 \mathrm{~d} 4 \mathrm{p}^{1} \mathrm{P}^{\circ}$ in $\mathrm{Ca}$ I and from the calculations of Burgess et al. (1995) in a non-exchange distorted-wave approximation for the transitions between the lowest seven terms (up to $4 \mathrm{f}^{2} \mathrm{~F}^{\circ}$ ) in Ca II. Uncertainties in data obtained by the $R$-matrix method are typically on the order of a few $10 \%$, and Samson \& Berrington note the excellent agreement of their results with experimentally derived rates for $4 s^{2}-4 s 4 p^{1} \mathrm{P}^{\circ}$. For the remaining bulk of the transitions, approximate formulae must be used. Basically, we apply the impact parameter method (IPM) of Seaton (1962a) to the allowed transitions and the Allen's formula (1973) with a collision strength of 1.0 to the optically forbidden transitions. Electronic collision rates based on Samson \& Berrington and Burgess et al. data where they are available, IPM data, and the Allen's formula for the remaining transitions are referred to below as the standard collisional recipe. For test purposes (see Sect. 3.3), we treat the allowed transitions absent in the Samson \& Berrington and Burgess et al. papers using the van Regemorter's formula (1962).

Electron impact ionization cross-sections are calculated by applying the formula of Seaton (1962b) with threshold photoionization cross-sections from the OP data.

\subsection{Model atmospheres. Programs and methodical notes}

Calcium is assumed to be a trace element because its contribution to the continuous opacity and the reservoir of free electrons is smaller by, at least, one order of magnitude than the contribution from the more abundant elements $\mathrm{Mg}, \mathrm{Si}$, and $\mathrm{Fe}$. Thus, we obtain statistical equilibrium populations for $\mathrm{Ca}$ I/II while keeping the atmospheric structure fixed. All calculations are performed with plane-parallel, homogeneous, LTE, and blanketed model atmospheres computed with the MAFAGS code (Fuhrmann et al. 1997).

We use a revised version of the DETAIL program (Butler \& Giddings 1985) based on the accelerated lambda iteration following the efficient method described by Rybicki \& Hummer $(1991,1992)$ in order to solve the coupled radiative transfer and statistical equilibrium equations. All bound-bound $(b-b)$ and bound-free $(b-f)$ transitions of Ca I and Ca II are explicitly taken into account in the SE calculations. The 15 strongest $b-b$ transitions in Ca I and all $L S J$ transitions $4 \mathrm{~s}-4 \mathrm{p}$ and $3 \mathrm{~d}-4 \mathrm{p}$ in Ca II are treated using the Voigt profile. Microturbulence is accounted for by inclusion of an additional term in the Doppler width. The van der Waals damping parameters based on the Anstee \& O'Mara's (1995) theory are taken from the VALD database (Kupka et al. 1999). The remaining $b-b$ transitions are treated using depth-dependent Doppler profiles.

In addition to the continuous background opacity, the line opacity introduced by both HI and metal lines is taken into 
Table 1. Atomic data for the isotopic components of Ca II $\lambda 8498$. The fractional isotope abundances $\epsilon$ correspond to solar-system matter.

\begin{tabular}{ccc}
\hline \hline$\lambda, \AA$ & isotope & $\log g f \epsilon$ \\
\hline 8498.023 & 40 & -1.43 \\
8498.079 & 42 & -3.60 \\
8498.106 & 43 & -4.29 \\
8498.131 & 44 & -3.10 \\
8498.179 & 46 & -5.81 \\
8498.223 & 48 & -4.14 \\
\hline
\end{tabular}

account by explicitly including it in solving the radiation transfer. The metal line list has been extracted from Kurucz' (1992) compilation and contains about 650000 atomic and molecular lines between $1300 \AA$ and $300000 \AA$. The Ca lines are excluded from the background.

The departure coefficients obtained from DETAIL are then used to compute the synthetic line profiles via the SIU program (www . usm. uni-muenchen. de/people/reetz/siu.html). In this step of the calculations, Voigt profile functions are adopted and the same microturbulence value $V_{\text {mic }}$ as in DETAIL is applied. Oscillator strengths and van der Waals damping constants of the $\mathrm{CaI}$ and $\mathrm{Ca}$ II lines and their accuracy are discussed in Sect. 4.

In the solar system's matter, $\mathrm{Ca}$ is represented by several isotopes with the isotope abundance ratio ${ }^{40} \mathrm{Ca}$ : ${ }^{42} \mathrm{Ca}:{ }^{43} \mathrm{Ca}:{ }^{44} \mathrm{Ca}:{ }^{46} \mathrm{Ca}:{ }^{48} \mathrm{Ca}=96.9: 0.647: 0.135: 2.09: 0.004: 0.187$ (Anders \& Grevesse 1989). In this study, we account for the isotope structure of the Ca II $\lambda 8498$ line $^{1}$ with the isotope shifts measured by Nörtershäuser et al. (1998). The wavelengths and the product of $g f$ and solar fractional isotope abundance $\epsilon$ are given in Table 1 for each isotope component. The oscillator strength is taken from the measurements of Theodosiou (1989), $\log g f(\lambda 8498)=-1.416$. Isotope shifts are much smaller $(<10 \mathrm{~m} \AA$ ) for the resonance lines in Ca I and Ca II (Lucas et al. 2004) and we treat them as single lines. No data is available for the remaining $\mathrm{Ca}$ lines. We neglect the hyperfine structure of $\mathrm{Ca}$ lines due to the very low fractional abundance of the only odd isotope ${ }^{43} \mathrm{Ca}(0.135 \%)$.

\section{NLTE effects as a function of stellar parameters}

In this section, we investigate the NLTE effects for Ca I/II in the following range of stellar parameters: $T_{\text {eff }}$ between $5000 \mathrm{~K}$ and $6000 \mathrm{~K}, \log g=3.0$ and 4.0 , and $[\mathrm{Fe} / \mathrm{H}]$ between 0 and -3 . Everywhere $V_{\text {mic }}=1 \mathrm{~km} \mathrm{~s}^{-1}$. For the models with $[\mathrm{Fe} / \mathrm{H}]=0$, the $\mathrm{Ca}$ abundance was assumed to follow the global metallicity. $\mathrm{Ca}$ enhancement with $[\mathrm{Ca} / \mathrm{Fe}]=0.4$ was assumed for the metal-poor models. Statistical equilibrium was computed using the standard recipe for electronic collisions and no hydrogenic collisions were taken into account $\left(S_{\mathrm{H}}=0\right)$.

\subsection{Statistical equilibrium of Ca I and NLTE effects for spectral lines}

Our calculations indicate a general behavior of departure coefficients, $b_{i}=n_{i}^{\mathrm{NLTE}} / n_{i}^{\mathrm{LTE}}$, independent of effective temperature, surface gravity, and metallicity. Here, $n_{i}^{\mathrm{NLTE}}$ and $n_{i}^{\mathrm{LTE}}$ are the statistical equilibrium and thermal (Saha-Boltzmann) number densities, respectively. The departure coefficients of the important

1 Of the three IR triplet lines, we look only at Ca II $\lambda 8498$, as $a$ ) it is the weakest of the three and $b$ ) it is least perturbed by Paschen lines.
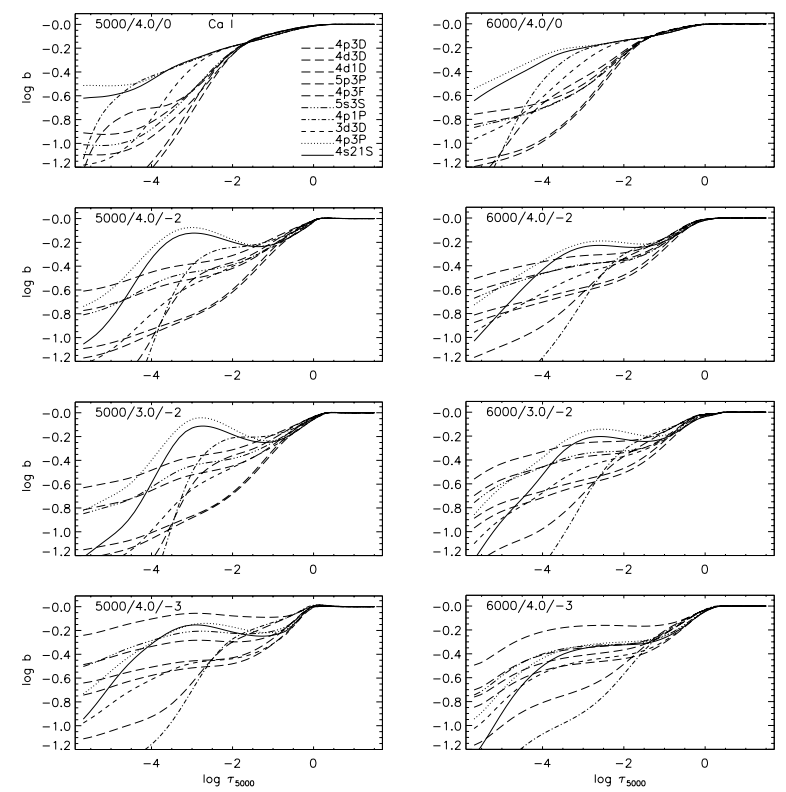

Fig. 2. Departure coefficients $\log b$ as a function of $\log \tau_{5000}$ for selected levels of Ca I, plotted for selected models of the grid. For each term of $3 \mathrm{~d}^{3} \mathrm{D}$ and $4 \mathrm{p}^{3} \mathrm{P}^{\circ}$, departure coefficients of the fine structure splitting sublevels are obtained to be close together and only the lowest sublevels, $3 \mathrm{~d}^{3} \mathrm{D}_{1}$ and $4 \mathrm{p}^{3} \mathrm{P}_{0}^{\circ}$, are shown. The stellar parameters are quoted in each panel.

levels of Ca I are plotted in Fig. 2 for selected models of our grid. As can be seen, all levels of Ca I are underpopulated in the atmospheric layers above $\log \tau_{5000}=0$. Overionization is caused by superthermal radiation of a non-local origin below the thresholds of the low excitation levels of Ca I. In atmospheres with solar or mildly deficient $\mathrm{Ca}$ abundance, $[\mathrm{Ca} / \mathrm{H}] \geq-1$, the most important levels are $4 \mathrm{p}^{1} \mathrm{P}^{\circ}, 3 \mathrm{~d}^{3} \mathrm{D}$, and $4 \mathrm{p}^{3} \mathrm{P}^{\circ}$ with the thresholds at $3898 \AA, 3450 \AA$, and $2930 \AA$, correspondingly. At the lower metallicity and $\mathrm{Ca}$ abundance, depopulation processes in $\mathrm{Ca} \mathrm{I}$ are dominated by enhanced ionization of the ground state due to a reduction of the continuous absorption coefficient below the threshold of this level at $2028 \AA$.

The statistical equilibrium of $\mathrm{Ca}$ I in the metallicity range between 0 and -1 is discussed in detail by Drake (1991). Referring to the main continuous opacity sources in the near ultraviolet wavelength regime, caused by the $\mathrm{H}^{-}$ions and excited neutral $\mathrm{H}$ atoms, Drake shows that overionization effects decrease with increasing model temperature, decreasing surface gravity, and decreasing $\mathrm{Ca}$ abundance. Our calculations for the stellar parameters overlapping with that of Drake support his conclusions qualitatively. However, quantitatively, overionization effects are found to be smaller in our work. The explanation lies with our extended model atom that contains energy levels up to $0.17 \mathrm{eV}$ below the ionization threshold, in contrast to $1.06 \mathrm{eV}$ in Drake's paper. Overionization effects on Ca I are partially cancelled due to collisional coupling of the Ca I high excitation levels to the ground state of the majority species, Ca II, that keeps thermodynamic equilibrium population. Our model atom provides this closer coupling and in this respect is more realistic than the one by Drake. The smaller departures from LTE for level populations result in weaker NLTE effects for spectral lines and smaller differences between derived NLTE and LTE Ca abundance, $\Delta_{\text {NLTE }}=\log \varepsilon_{\text {NLTE }}-\log \varepsilon_{\text {LTE }}$. We refer to $\Delta_{\text {NLTE }}$ as the NLTE abundance correction. For example, for atmospheric parameters $T_{\text {eff }}=5800 \mathrm{~K}, \log g=4.5,[\mathrm{Fe} / \mathrm{H}]=0$, Drake gives 

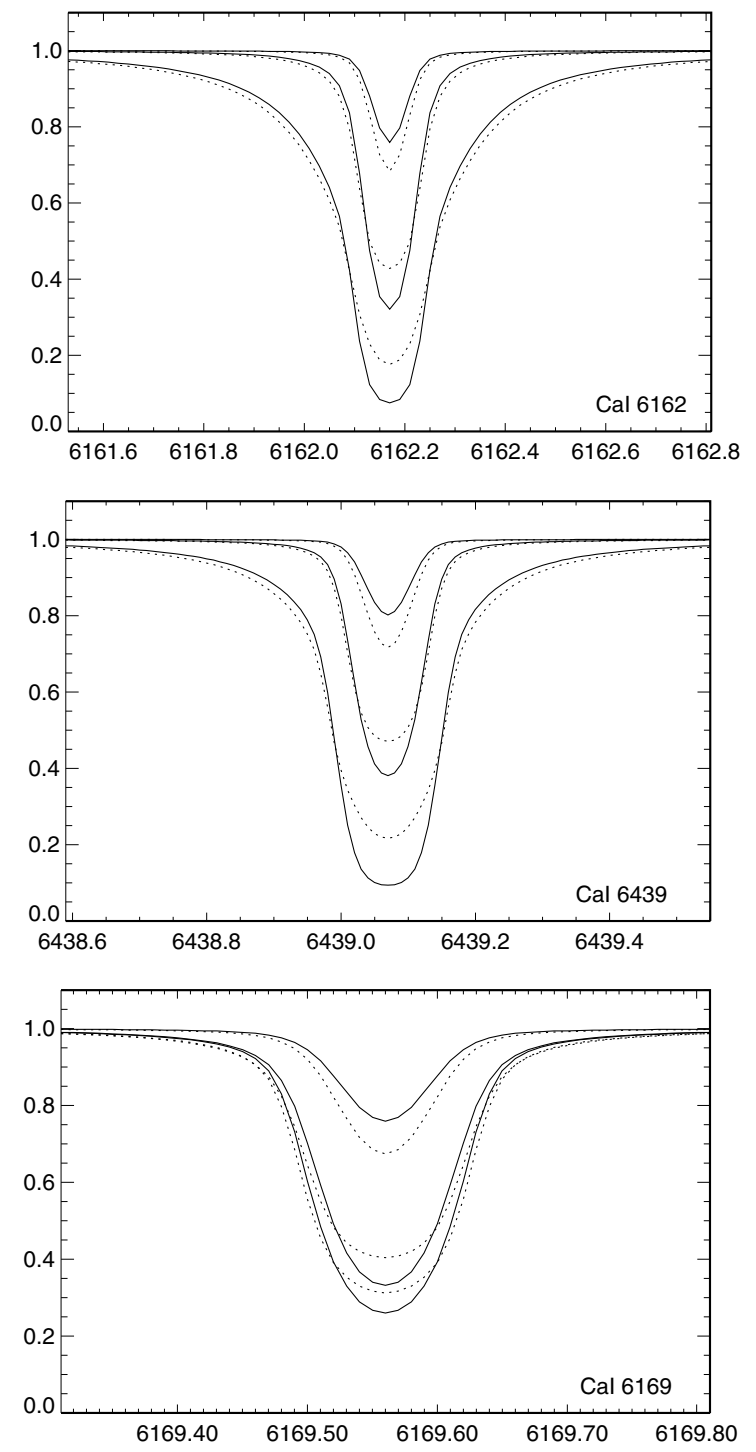

Fig. 3. NLTE (continuous line) and LTE (dotted line) theoretical profiles of the selected Ca I lines for the models with the same $T_{\text {eff }}=5500 \mathrm{~K}$ and $\log g=4.0$ but with different metallicities. For the $\lambda 6162$ and $\lambda 6439$ lines, $[\mathrm{Fe} / \mathrm{H}]=0,-2$, and -3 from bottom to top; for $\lambda 6169$, $[\mathrm{Fe} / \mathrm{H}]=0,-1$, and -2 .

nearly equal values $\Delta_{\mathrm{NLTE}}=0.11$ dex for $\lambda 5261$ (multiplet 22), $\lambda 6455$ (multiplet 19), and $\lambda 6166$ (multiplet 20). The corresponding values from our calculations are $0.04 \mathrm{dex}, 0.05 \mathrm{dex}$, and 0.07 dex.

In contrast to the models with $[\mathrm{Fe} / \mathrm{H}] \geq-1$, overionization effects increase with increasing model temperature and decreasing global metallicity, when ones goes to the lower metallicity and $\mathrm{Ca}$ abundance, $[\mathrm{Fe} / \mathrm{H}]$ and $[\mathrm{Ca} / \mathrm{H}]<-1$. The explanation lies with the behavior of the continuous absorption coefficient below $2028 \AA$ that is, in metal-poor atmospheres, mainly due to quasi-molecular hydrogen absorption (Doyle 1968), the $\mathrm{H}^{-}$ion, and the $\mathrm{H}_{2}^{+}$ion. The contribution of the $\mathrm{H}^{-}$ion increases with increasing effective temperature and decreases with decreasing metallicity, while the contribution of quasi-molecular hydrogen absorption changes in the opposite direction. Their competition results in the effects seen in Fig. 2.

The NLTE effects on spectral lines are illustrated in Fig. 3 and Table 2. Figure 3 shows the NLTE and LTE profiles of three representative lines of neutral calcium computed for the models with a different global metal abundance. These are (i) the low excitation line $\lambda 6162$, which is the strongest among all subordinate lines; (ii) $\lambda 6439$ with the largest oscillator strength, $f_{i j}=0.390$, but relatively small van der Waals damping constant $\log C_{6}=-31.58$, which is lower by 1.28 dex than that of $\lambda 6162$; and (iii) the intermediate strength line $\lambda 6169.5$. Table 2 presents NLTE abundance corrections, $\Delta_{\text {NLTE }}$, for $16 \mathrm{Ca}$ I lines. Three other Ca I lines, $\lambda 6161, \lambda 6169.0$, and $\lambda 6122$, have $\Delta_{\text {NLTE }}$ close to those of $\lambda 6166, \lambda 6169.5$, and $\lambda 6162$, respectively. The NLTE and LTE line profiles and equivalent widths were calculated with the laboratory oscillator strengths and $C_{6}$ values given in Table 4 . The microturbulence value, $V_{\text {mic }}=1 \mathrm{~km} \mathrm{~s}^{-1}$, was adopted for all the models. Departures from LTE for spectral lines can be understood by considering the depths of formation of various parts of the lines and by inspecting the level departure coefficients and line source functions. In the metallicity regime, $[\mathrm{Fe} / \mathrm{H}] \geq-1$, our results support the conclusions of Drake (1991): for each investigated line, NLTE effects lead to enhanced absorption in the line core and depleted absorption in the line wings (Fig. 3). This can be understood because the line wings are formed in deep layers where overionization depopulates all Ca I levels, but the line cores are formed at small depths, above $\log \tau_{5000}=-2$, where the upper levels of the transitions are underpopulated to a greater extent than are the lower levels due to photon losses in the line wings. The most prominent example is a steep decrease in $b\left(4 \mathrm{p}^{1} \mathrm{P}^{\circ}\right)$ above $\log \tau_{5000}=-2$ in all metal-poor models (Fig. 2). The line source function drops below the Planck function at these depths resulting in the enhanced absorption in the line cores. The combined effect on the line strength is that the NLTE abundance correction is small in most cases. Its sign and value depend on the contributions of the line core and wings to the overall line strength. The NLTE corrections tend toward negative values with increasing $T_{\text {eff }}$ and decreasing $\log g$. They are more negative for the lines of multiplet 18, $\lambda 6439, \lambda 6471$, $\lambda 6493$, and $\lambda 6499$ (Table 2). All these trends reflect the behavior of the van der Waals broadened line wings.

In contrast to the solar metallicity models, the energy levels become weakly coupled far inside the metal-poor atmospheres with $[\mathrm{Fe} / \mathrm{H}]<-1$ due to deficient collisions (Fig. 2). For each model, at the depths where the weak lines are formed, the upper levels are all depleted to a lesser extent relative to their LTE populations than are the lower levels. The lines are weaker relative to their LTE strengths not only due to the general overionization but also due to $b_{\mathrm{u}} / b_{1}>1$ resulting in the line source function $S_{\text {lu }} \simeq b_{\mathrm{u}} / b_{1} B_{v}>B_{v}$ and the depleted line absorption. Here, $b_{\mathrm{u}}$ and $b_{1}$ are the departure coefficients for the upper and lower levels of the transition. For example, this is valid for $\lambda 6169.5$ in the model with $T_{\text {eff }}=5500 \mathrm{~K}, \log g=4.0,[\mathrm{Fe} / \mathrm{H}]=-2$ and $\lambda 6162$ and $\lambda 6439$ in the model $5500 \mathrm{~K} / 4.0 /-3$. The corresponding NLTE and LTE profiles are shown in Fig. 3. If the line is strong for a given set of stellar parameters (e.g., $\lambda 6162$ and $\lambda 6439$ for the models with $[\mathrm{Fe} / \mathrm{H}]=-2)$, the NLTE effects on line profiles are similar to the effects for the models with $[\mathrm{Fe} / \mathrm{H}] \geq-1$.

\subsection{Statistical equilibrium of Ca II and NLTE effects on spectral lines}

Figure 4 shows the departure coefficients for some important levels of Ca II plotted for selected models of our grid. In the temperature regime we are concerned with here $\left(T_{\text {eff }}=\right.$ $5000 \mathrm{~K}-6000 \mathrm{~K}$ ), Ca II dominates the element number density over atmospheric depths. Thus, no process seems to affect the Ca II ground-state population, and $4 \mathrm{~s}$ keeps its thermodynamic equilibrium value. An exception is the uppermost 
Table 2. NLTE abundance corrections* (dex) for the Ca I and Ca II lines depending on effective temperature, surface gravity, and metallicity as computed by ignoring hydrogenic collisions $\left(S_{\mathrm{H}}=0\right)$ in SE calculations.

\begin{tabular}{|c|c|c|c|c|c|c|c|c|c|c|c|c|c|c|c|c|c|c|c|}
\hline \multirow{2}{*}{$\begin{array}{c}T_{\text {eff }} / \log g / \\
{[\mathrm{Fe} / \mathrm{H}]}\end{array}$} & \multicolumn{16}{|c|}{$\overline{\mathrm{Ca} I}$} & \multicolumn{3}{|c|}{$\overline{\mathrm{Ca} \text { II }}$} \\
\hline & 4226 & 4425 & 5261 & 5349 & 5512 & 5588 & 5590 & 5857 & 5867 & 6162 & 6166 & 6169 & 6439 & 6471 & 6493 & 6499 & 8498 & 8248 & 8927 \\
\hline $5780 / 4.44 / 0$ & 0.07 & 0.04 & 0.04 & 0.01 & 0.0 & 0.01 & 0.03 & 0.00 & 0.06 & 0.01 & 0.07 & .02 & -0.04 & -0.04 & -0.04 & -0.01 & -0.02 & -0.11 & -0.19 \\
\hline $5000 / 3 / 0$ & 11 & 8 & 0.1 & c & 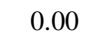 & 07 & .12 & 0.00 & 0.04 & 0.07 & 0.12 & 0.05 & & 0.00 & 0.05 & 0.04 & .02 & -0.12 & -0.13 \\
\hline $5000 / 3 /-2$ & 0.24 & 0.17 & 0.35 & 0.35 & 0.10 & 0.07 & 0.26 & 0.09 & 0.08 & 0.00 & 0.30 & 0.24 & -0.04 & 0.14 & 0.17 & 0.20 & -0.08 & & -0.11 \\
\hline $5000 / 3 /-3$ & 0.24 & 0.32 & & & & 0.32 & & 0.20 & & 0.17 & & & 0.27 & & 0.30 & & -0.13 & & \\
\hline $5000 / 4 / 0$ & 0.09 & 0.08 & 0.08 & 0.05 & 0.03 & 0.07 & 0.08 & 0.04 & 0.08 & 0.07 & 0.09 & 0.06 & 0.06 & 0.01 & 0.05 & 0.03 & -0.02 & -0.07 & -0.14 \\
\hline $5000 / 4 /-1$ & 0.14 & 0.12 & 0.13 & 0.07 & 0.06 & 0.08 & 0.11 & 0.03 & 0.11 & 0.07 & 0.16 & 0.08 & 0.01 & 0.00 & 0.02 & 0.04 & -0.03 & -0.05 & -0.13 \\
\hline $5000 / 4 /-3$ & 0.23 & 0.25 & & & & 0.27 & & 0.12 & & 0.16 & & & 0.22 & & 0.24 & & -0.16 & & \\
\hline 5500 & 0.10 & 0.05 & 0.07 & 0.00 & -0.03 & -0.02 & 0.06 & -0.07 & 0.02 & -0.02 & 0.10 & -0.02 & -0.11 & -0.05 & -0.06 & 0.00 & -0.02 & -0.20 & -0.22 \\
\hline $5500 / 3 /-2$ & 0.20 & 0.19 & 0.32 & 0.27 & 0.12 & 0.10 & 0.25 & 0.13 & 0.11 & -0.04 & 0.28 & 0.23 & -0.02 & 0.14 & 0.19 & 0.18 & -0.11 & -0.02 & -0.16 \\
\hline $5500 / 3 /-3$ & 0.12 & 0.29 & & & & & & 0.24 & & & & & & & 0.26 & & & & \\
\hline 5500 & 0.08 & 0.05 & 0.05 & 0.0 & 0.01 & 0.03 & 0.05 & -0.01 & 0.05 & 0.03 & 0.08 & 0.02 & -0.02 & -0.04 & -0.02 & 0.00 & -0.02 & -0.10 & -0.13 \\
\hline $5500 / 4 /-1$ & 0.13 & 0.08 & 0.14 & 0.08 & 0.06 & 0.00 & 0.09 & 0.00 & 0.10 & 0.00 & 0.15 & 0.06 & -0.10 & -0.01 & -0.04 & 0.04 & -0.03 & -0.09 & -0.21 \\
\hline $5500 / 4 /-2$ & 0.1 & 0.18 & 0.28 & 0.25 & 0.11 & 0.10 & 0.22 & 0.13 & 0.11 & 0.03 & 0.25 & 0.21 & 0.00 & 0.12 & 0.16 & 0.15 & -0.08 & & -0.10 \\
\hline $6000 / 3 /-2$ & 0.10 & 0.20 & 0.26 & 0.21 & 0.13 & 0.13 & 0.22 & 0.16 & & -0.01 & & 0.21 & 0.01 & 0.11 & 0.17 & 0.14 & -0.16 & -0.04 & -0.20 \\
\hline $6000 / 3 /-3$ & 0.02 & 0.26 & & & & 0.27 & & & & 0.19 & & & 0.22 & & & & -0.41 & & 0.00 \\
\hline $6000 / 4 / 0$ & 0.07 & 0.02 & 0.04 & 0.00 & 0.01 & -0.05 & 0.01 & -0.05 & 0.05 & -0.05 & 0.06 & -0.01 & -0.13 & -0.06 & -0.10 & -0.02 & -0.02 & -0.17 & -0.27 \\
\hline $6000 / 4 /-1$ & 0.11 & 0.03 & 0.13 & 0.08 & 0.05 & -0.10 & 0.07 & -0.02 & 0.07 & -0.10 & 0.13 & 0.05 & -0.22 & -0.01 & -0.08 & 0.03 & -0.05 & -0.13 & -0.29 \\
\hline $6000 / 4 /-2$ & 0.13 & 0.18 & 0.24 & 0.20 & 0.12 & 0.12 & 0.19 & 0.15 & & 0.03 & & 0.19 & 0.01 & 0.10 & 0.14 & 0.13 & -0.11 & -0.01 & -0.12 \\
\hline $6000 / 4 /-3$ & 0.10 & 0.25 & & & & 0.29 & & & & 0.19 & & & 0.21 & & & & -0.23 & & \\
\hline
\end{tabular}

* Blanks denote theoretical NLTE equivalent widths below $5 \mathrm{~m} \AA$.
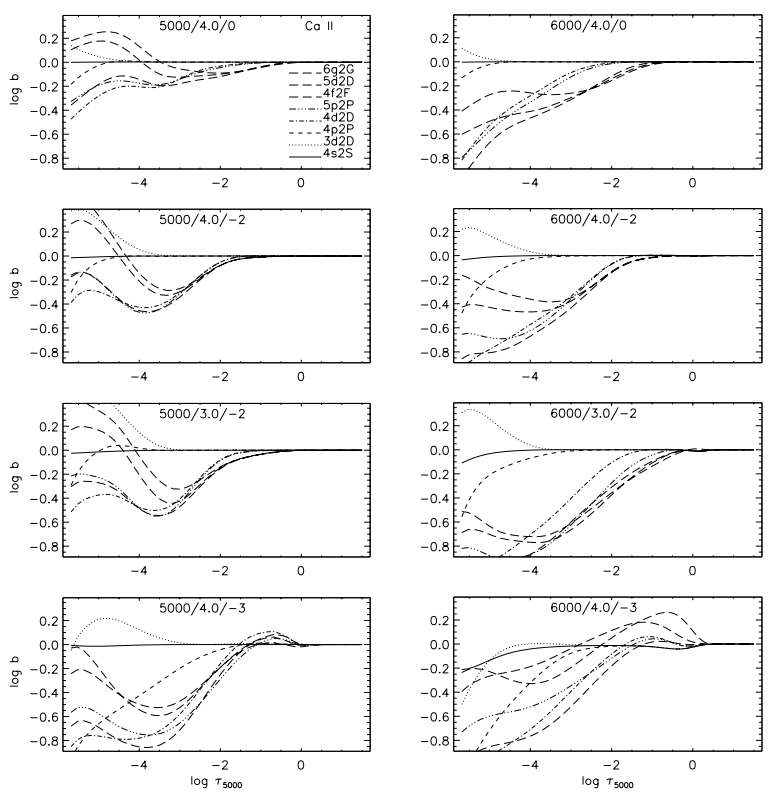

Fig. 4. The same as in Fig. 2 for Ca II.

layers above $\log \tau_{5000}=-4$ in the very metal-poor $([\mathrm{Fe} / \mathrm{H}] \leq$ -2) models with $T_{\text {eff }}=6000 \mathrm{~K}$. Ca II competes there with Ca III in contributing to the element population, and enhanced ionization of the low excitation levels of Ca II leads to underpopulation of the total Ca II. This effect is amplified with decreasing metallicity. The levels $3 \mathrm{~d}$ and $4 \mathrm{p}$ follow the ground state in deep layers, and their coupling is lost at the depths where, for each model, photon losses in the weakest line $\lambda 8498$ of the multiplet $3 \mathrm{~d}-4 \mathrm{p}$ start to become important. These are the uppermost layers above $\log \tau_{5000}=-5$ in the solar metallicity models and above $\log \tau_{5000}=-3$ in the models with $[\mathrm{Fe} / \mathrm{H}]=-2$. At $[\mathrm{Fe} / \mathrm{H}]=-3$, detailed balance in the transition $3 \mathrm{~d}-4 \mathrm{p}$ is destroyed in the deeper layers around $\log \tau_{5000}=-2$. The departure coefficients of $4 \mathrm{~d}^{2} \mathrm{D}$ and the higher excitation levels begin to deviate from 1 far inside the atmosphere even in the solar metallicity models due to photon losses in the transitions to low excitation levels.

NLTE leads to strengthened Ca II lines and negative NLTE abundance corrections. The behavior of individual lines is as follows.

In the stellar parameter range we are concerned with in this paper, departures from LTE occur only in the very core of Ca II K $\lambda$ 3933. The NLTE abundance correction does not exceed 0.02 dex in absolute value. This can be understood because the line continues to be strong even at $[\mathrm{Ca} / \mathrm{H}]=-3$, and its line strength is dominated by the line wings.

Similar to the resonance line, the IR lines of multiplet $3 d-4 p$, $\lambda 8498, \lambda 8542$, and $\lambda 8662$, reveal NLTE effects only in the Doppler core. The line core is strengthened because the line source function drops below the Planck function due to $b_{\mathrm{u}} / b_{1}<1$ and the lower level of the transition is overpopulated $\left(b_{1}>1\right)$ at the line core formation depths. The NLTE correction is larger in absolute value for the weakest line, $\lambda 8498$, compared to the other two due to a smaller contribution from the line wings to the overall line strength. However, even for $\lambda 8498, \Delta_{\mathrm{NLTE}}$ is small for the models with $[\mathrm{Fe} / \mathrm{H}]=0$ and $-1,\left|\Delta_{\mathrm{NLTE}}\right| \leq 0.05$ (Table 2). The van der Waals broadened wings are weakened with decreasing $\mathrm{Ca}$ abundance and, at a fixed metallicity, with decreasing surface gravity, and increasing temperature. The NLTE effects are amplified in the same directions such that $\Delta_{\text {NLTE }}(\lambda 8498)$ reaches $-0.41 \mathrm{dex}$ for the model $6000 \mathrm{~K} / 3.0 /-3$. Significant NLTE effects can be expected in the low-density atmospheres of metal-poor red giants. Further investigations will be conducted when we have proper observations at our disposal.

The IR high excitation lines of multiplets $5 \mathrm{p}-5 \mathrm{~d}, \lambda 8248$ and $\lambda 8254$, and 4d-4f, $\lambda 8912$ and $\lambda 8927$, can be good candidates for determining the Ca II abundance in close to solar-metallicity 
Table 3. Uncertainties in the NLTE analysis of Ca I/II, where blanks denote NLTE theoretical equivalent widths below $5 \mathrm{~mA}$.

\begin{tabular}{|c|c|c|c|c|c|c|c|c|c|}
\hline \multirow[b]{2}{*}{ input parameter } & \multicolumn{9}{|c|}{ changes in NLTE abundance corrections (dex) } \\
\hline & Ca I 4226 & 4425 & 5588 & 5857 & 6162 & 6169 & 6439 & Ca II 8498 & 8927 \\
\hline & $5500 \mathrm{~K}$ & $/ 4.0 /-$ & & & & & & & \\
\hline Photoionization: & -0.02 & -0.02 & -0.05 & -0.06 & -0.03 & -0.04 & -0.06 & 0.00 & 0.00 \\
\hline$e$-collisions: & -0.02 & -0.01 & -0.03 & 0.01 & 0.00 & -0.02 & -0.02 & 0.00 & 0.14 \\
\hline H collisions: & -0.12 & -0.08 & -0.05 & -0.03 & -0.04 & -0.08 & -0.04 & 0.01 & 0.16 \\
\hline$\Delta V_{\mathrm{mic}}=+0.5 \mathrm{~km} \mathrm{~s}^{-1}$ & 0.00 & -0.01 & -0.02 & 0.00 & -0.02 & 0.00 & -0.03 & 0.00 & 0.01 \\
\hline & $5500 \mathrm{~K}$ & / $4.0 /-$ & & & & & & & \\
\hline Photoionization: & -0.01 & -0.02 & -0.02 & -0.03 & -0.01 & -0.01 & -0.03 & 0.00 & 0.00 \\
\hline$e$-collisions: & -0.04 & -0.01 & -0.03 & 0.04 & 0.00 & -0.01 & -0.02 & 0.02 & 0.08 \\
\hline H collisions: & -0.18 & -0.17 & -0.12 & -0.10 & -0.06 & -0.19 & -0.10 & 0.03 & 0.09 \\
\hline$\Delta V_{\mathrm{mic}}=+0.5 \mathrm{~km} \mathrm{~s}^{-1}$ & 0.00 & 0.00 & 0.01 & 0.00 & 0.00 & 0.00 & 0.01 & -0.01 & 0.00 \\
\hline & $5500 \mathrm{~K}$ & $/ 4.0 /-$ & & & & & & & \\
\hline Photoionization: & -0.01 & -0.01 & -0.01 & -0.02 & -0.01 & & -0.01 & 0.00 & \\
\hline$e$-collisions: & -0.03 & -0.01 & -0.03 & 0.02 & 0.00 & & -0.01 & 0.07 & \\
\hline H collisions: & -0.19 & -0.20 & -0.21 & -0.12 & -0.10 & & -0.18 & 0.10 & \\
\hline$\Delta V_{\text {mic }}=+0.5 \mathrm{~km} \mathrm{~s}^{-1}$ & -0.01 & 0.00 & 0.00 & 0.00 & 0.00 & & 0.00 & -0.03 & \\
\hline
\end{tabular}

cool stars. They are of intermediate strength and nearly free of blends. It can be seen from Fig. 4 that the high excitation levels are all depleted relative to their LTE populations at line formation depths in the models with $[\mathrm{Fe} / \mathrm{H}] \geq-2$. However, the lower levels of the considered transitions, $5 \mathrm{p}$ and $4 \mathrm{~d}$, are underpopulated to a lesser extent than are the upper levels, 5d and 4f, resulting in smaller line source functions compared to the Planck function and enhanced line absorption. The NLTE effects are significant even for the solar metallicity models and become stronger with increasing $T_{\text {eff }}$ and decreasing $\log g$. The lines of multiplet $5 \mathrm{p}-5 \mathrm{~d}$ are weaker compared to $\lambda 8912$ and $\lambda 8927$ and, in general, reveal smaller NLTE effects. In Table 2, we present $\Delta_{\text {NLTE }}$ for $\lambda 8248$ and $\lambda 8927$. The NLTE corrections for the second line of multiplet $4 \mathrm{~d}-4 \mathrm{f}$ are close to the corresponding values for $\lambda 8927$ and, in general, are smaller by up to $0.03 \mathrm{dex}$ in absolute value. For $\lambda 8254$, the NLTE effects are very small with $\Delta_{\text {NLTE }}$ at the level of a few parts in a hundred.

\subsection{Error estimates for the Ca I/II SE calculations}

To assess the effects of crucial atomic data on the accuracy of NLTE $\mathrm{Ca}$ abundances derived from the $\mathrm{Ca}$ I and $\mathrm{Ca}$ II lines, test calculations were performed for three models with $T_{\text {eff }}=$ $5500 \mathrm{~K}, \log g=4.0$, and $[\mathrm{Fe} / \mathrm{H}]=-1,-2$, and -3 . For each parameter or set of cross-sections that we varied, we computed a small grid for a given line at different abundances to determine the systematic shift in $\mathrm{Ca}$ abundance needed to fit the NLTE equivalent width evaluated using our standard set of atomic data. The results of the tests are summarized for selected lines in Table 3.

The OP photoionization data based on the R-matrix calculations are typically accurate to $10 \%$. In test computations, we assumed a factor of two uncertainty of photoionization crosssections as a worst case. As expected, a variation in the photoionization rates affects the lines of the minority species ( $\mathrm{Ca}$ I) by way of a displaced ionization balance and does not affect the lines of the majority species (Ca II). Corrections for the Ca I lines decrease with decreasing metallicity. The explanation lies with an increasing fraction of Ca III at the same optical depth in the models with decreasing metallicity. When cross-sections of both the $\mathrm{Ca}$ I and $\mathrm{Ca}$ II levels change, the $\mathrm{Ca}$ I/Ca II/Ca III ionization balance is established in such way that the $\mathrm{Ca} / \mathrm{Ca}$ II ratio is influenced to a lesser extent in the more metal-poor models.
In the present work, detailed electronic collision excitation data are used for a considerable number of transitions in Ca II and for all important transitions from the ground state in Ca I. In test calculations, we varied collisional rates for the remaining transitions applying the van Regemorter's formula (1962) instead of IPM data. Van Regemorter's collisional rates $C_{i j}(\mathrm{Reg})$ are, in general, higher than the corresponding IPM-based values, by up to 2 orders of magnitude for the transitions with energy separation $\Delta E<2 \mathrm{eV}$ (see Fig. 2 in Mashonkina 1996, for the transitions in $\mathrm{MgI}$ ). As a result, NLTE effects are weakened compared to the standard collisional recipe, and a NLTE $\mathrm{Ca}$ abundance is obtained to be closer to the LTE one (lower from the $\mathrm{Ca}$ I lines and higher from the $\mathrm{Ca}$ II lines, Table 3 ). The only exception is Ca I 5857 for which NLTE effects are strengthened. In any case, a variation in the electronic collision rates only weakly affects the $\mathrm{Ca}$ I lines, such that the derived $\mathrm{Ca}$ abundance changes by $0.04 \mathrm{dex}$, at maximum. In contrast, the effect is significant for the $\mathrm{Ca}$ II lines, and a change in $\mathrm{Ca}$ abundance may consist of $0.07 \mathrm{dex}$ to $0.14 \mathrm{dex}$ for different lines. A different reaction of the $\mathrm{Ca} I$ and $\mathrm{Ca}$ II lines can be understood because the main mechanism of departures from LTE is connected with $b-f$ transitions for Ca I, while with $b-b$ transitions for Ca II.

We also inspected the effect of including hydrogenic collisions in our SE calculations. Table 3 shows the difference in Ca abundance derived assuming $S_{\mathrm{H}}=1$ and ignoring $\mathrm{H}$ collisions $\left(S_{\mathrm{H}}=0\right)$. As expected, departures from LTE are weakened for the Ca II lines. Changes in Ca II abundance are comparable to those obtained when varying electronic collision rates. A somewhat unexpected behavior is seen for most $\mathrm{Ca}$ I lines in the model with $[\mathrm{Fe} / \mathrm{H}]=-1$ and for $\lambda 6439$ in the model with $[\mathrm{Fe} / \mathrm{H}]=-2$ : NLTE effects seem to be amplified when the total collisional rates increase. This can be understood because departures from LTE go down in the line wings, as expected, but are hardly changed in the cores of strong lines due to the inefficiency of collisions in the uppermost atmospheric layers. Thus, the line wings act no longer to reduce the combined NLTE effect on the overall line strength, and it becomes larger compared to the case of electronic collisions alone.

In addition, we examined the effect of a shift of the line formation depths due to increasing the microturbulence value in the model. An increase in $V_{\text {mic }}$ by $0.5 \mathrm{~km} \mathrm{~s}^{-1}$ has a negligible effect on $\Delta_{\text {NLTE }}$ for every Ca line in the models with $[\mathrm{Fe} / \mathrm{H}]=-2$ and -3 and leads to strengthening NLTE effects for 
Table 4. Atomic data for the Ca I and Ca II lines, solar $\log g f \varepsilon_{\mathrm{Ca}}^{\odot}$ values determined from NLTE analysis of the Ca line profiles in the Kitt Peak Solar Atlas (Kurucz et al. 1984) neglecting hydrogenic collisions $\left(S_{\mathrm{H}}=0\right)$, and abundance corrections $\Delta_{\mathrm{X}}=\log \varepsilon_{\mathrm{X}}-\log \varepsilon_{\mathrm{S}_{\mathrm{H}}=0}$ where $\mathrm{X}$ takes the meaning $0.1,1$, and LTE for $S_{\mathrm{H}}=0.1, S_{\mathrm{H}}=1$ and the LTE assumption, respectively.

\begin{tabular}{|c|c|c|c|c|c|c|c|c|c|c|c|c|}
\hline \multirow{2}{*}{$\begin{array}{c}\lambda \\
(\AA)\end{array}$} & \multirow[t]{2}{*}{ mult } & \multirow{2}{*}{$\begin{array}{l}E_{\text {low }} \\
(\mathrm{eV})\end{array}$} & \multicolumn{4}{|c|}{$\log g f$} & \multirow[t]{2}{*}{$\log C_{6}$} & \multirow{2}{*}{$\log g f \varepsilon_{\mathrm{Ca}}^{\odot}$} & \multirow[t]{2}{*}{$\Delta_{0.1}$} & \multirow{2}{*}{$\Delta_{1}$} & \multirow{2}{*}{$\overline{\Delta_{\mathrm{LTE}}}$} & \multirow{2}{*}{$\begin{array}{c}W_{\lambda} \\
(\mathrm{m} \AA)\end{array}$} \\
\hline & & & LAB & NIST & VALD & $\mathrm{OP}$ & & & & & & \\
\hline 1 & 2 & 3 & 4 & 5 & 6 & 7 & 8 & 9 & 10 & 11 & 12 & 13 \\
\hline \multicolumn{13}{|c|}{$\mathrm{Ca} I$} \\
\hline 4226.73 & 2 & 0.00 & $0.244^{a}$ & 0.244 & 0.265 & 0.276 & $-31.23^{g}$ & -5.47 & -0.03 & -0.06 & -0.08 & 1500 \\
\hline 4425.44 & 4 & 1.87 & $-0.358^{b}$ & -0.358 & -0.286 & -0.566 & $-30.90^{*}$ & -5.95 & 0.00 & -0.03 & -0.03 & 174 \\
\hline 4578.56 & 23 & 2.51 & $-0.697^{c}$ & -0.558 & -0.697 & -0.804 & $-30.30^{i}$ & -6.33 & 0.00 & -0.04 & -0.06 & 87 \\
\hline 5261.71 & 22 & 2.51 & $-0.579^{c}$ & -0.73 & -0.579 & -0.836 & $-30.86^{i}$ & -6.14 & 0.00 & -0.03 & -0.05 & 102 \\
\hline 5349.47 & 33 & 2.70 & $-0.310^{c}$ & - & -0.310 & -0.730 & $-31.45^{i}$ & -5.92 & 0.02 & 0.02 & 0.00 & 97 \\
\hline $5512.98^{1}$ & & 2.92 & $-0.464^{d}$ & -0.30 & -0.447 & -0.396 & $-30.61^{i}$ & -6.05 & 0.00 & 0.02 & 0.00 & 89 \\
\hline 5588.76 & 21 & 2.51 & $0.358^{c}$ & 0.21 & 0.358 & 0.339 & $-31.39^{i}$ & -5.36 & 0.00 & 0.00 & 0.00 & 171 \\
\hline 5590.12 & 21 & 2.51 & $-0.571^{c}$ & -0.71 & -0.571 & -0.590 & $-31.39^{i}$ & -6.18 & 0.00 & -0.03 & -0.03 & 97 \\
\hline 5857.45 & 47 & 2.92 & $0.240^{c}$ & 0.23 & 0.240 & 0.025 & $-30.61^{i}$ & -5.40 & 0.00 & -0.02 & -0.02 & 160 \\
\hline $5867.57^{2}$ & & 2.92 & $-1.570^{d}$ & - & -0.801 & -2.402 & $-30.97^{h}$ & -7.12 & -0.02 & -0.06 & -0.09 & 26 \\
\hline 6122.22 & 3 & 1.88 & $-0.315^{b}$ & -0.315 & -0.386 & -0.356 & $-30.30^{g}$ & -6.06 & 0.01 & -0.01 & -0.02 & 228 \\
\hline 6162.17 & 3 & 1.89 & $-0.089^{b}$ & -0.089 & -0.167 & -0.137 & $-30.30^{g}$ & -5.83 & 0.02 & 0.00 & -0.02 & 289 \\
\hline 6161.29 & 20 & 2.51 & $-1.266^{c}$ & -1.03 & -1.266 & -1.290 & $-30.48^{i}$ & -6.85 & -0.01 & -0.06 & -0.07 & 67 \\
\hline 6166.44 & 20 & 2.51 & $-1.142^{c}$ & -0.90 & -1.142 & -1.166 & $-30.48^{i}$ & -6.70 & -0.02 & -0.06 & -0.07 & 77 \\
\hline 6169.06 & 20 & 2.51 & $-0.797^{c}$ & -0.54 & -0.797 & -0.821 & $-30.48^{i}$ & -6.40 & -0.02 & -0.03 & -0.03 & 100 \\
\hline 6169.56 & 20 & 2.51 & $-0.478^{c}$ & -0.27 & -0.478 & -0.502 & $-30.48^{i}$ & -6.09 & -0.02 & -0.03 & -0.03 & 129 \\
\hline 6439.07 & 18 & 2.51 & $0.390^{c}$ & 0.47 & 0.390 & 0.301 & $-31.58^{i}$ & -5.32 & -0.02 & -0.01 & -0.01 & 188 \\
\hline 6471.66 & 18 & 2.51 & $-0.686^{c}$ & -0.59 & -0.686 & -0.775 & $-31.58^{i}$ & -6.33 & -0.02 & 0.01 & 0.01 & 97 \\
\hline 6493.78 & 18 & 2.51 & $-0.109^{c}$ & 0.14 & -0.109 & -0.198 & $-31.58^{i}$ & -5.77 & -0.03 & -0.01 & 0.00 & 139 \\
\hline 6499.65 & 18 & 2.51 & $-0.818^{c}$ & -0.59 & -0.818 & -0.907 & $-31.58^{i}$ & -6.41 & -0.03 & -0.06 & 0.00 & 89 \\
\hline 6449.81 & 19 & 2.51 & $-0.502^{c}$ & -0.55 & -0.502 & - & $-31.45^{i}$ & -6.14 & -0.02 & 0.00 & 0.00 & 106 \\
\hline 6455.60 & 19 & 2.51 & $-1.34^{d}$ & -1.36 & -1.29 & - & $-31.45^{i}$ & -6.95 & -0.02 & -0.04 & -0.05 & 59 \\
\hline 6572.78 & 1 & 0.00 & $-4.24^{e}$ & -4.30 & -4.104 & - & $-31.54^{g}$ & -9.90 & -0.03 & -0.08 & -0.10 & 31 \\
\hline 7326.15 & 44 & 2.92 & $-0.208^{d}$ & - & 0.073 & 0.021 & $-31.42^{h}$ & -5.74 & 0.02 & 0.00 & 0.02 & 116 \\
\hline \multicolumn{13}{|c|}{$\mathrm{Ca}$ II } \\
\hline 5339.19 & 20 & 8.40 & - & - & -1.099 & -0.079 & $-30.31^{h}$ & -5.77 & 0.00 & 0.00 & 0.00 & 6 \\
\hline 5001.48 & 15 & 7.47 & - & -0.52 & -0.755 & -0.505 & $-30.66^{h}$ & -6.18 & 0.00 & 0.01 & 0.03 & 11 \\
\hline 6456.91 & 19 & 8.40 & - & - & -0.171 & 0.412 & $-30.77^{h}$ & -5.20 & 0.01 & 0.02 & 0.02 & 16 \\
\hline 8248.80 & 13 & 7.48 & - & 0.57 & 0.621 & 0.556 & $-30.85^{*}$ & -4.97 & 0.02 & 0.08 & 0.14 & 65 \\
\hline 8254.70 & 13 & 7.48 & - & -0.39 & -0.333 & -0.398 & $-30.85^{*}$ & -5.92 & 0.00 & 0.01 & 0.02 & 18 \\
\hline 8498.02 & 2 & 1.69 & $-1.416^{f}$ & -1.318 & -1.416 & -1.465 & $-31.51^{g}$ & -7.14 & 0.00 & 0.00 & 0.00 & 1132 \\
\hline $8912.07^{3}$ & & 7.05 & - & - & 0.575 & 0.636 & $-31.10^{*}$ & -4.98 & 0.03 & 0.10 & 0.17 & 111 \\
\hline $8927.36^{4}$ & & 7.05 & - & - & 0.750 & 0.811 & $-31.10^{*}$ & -4.81 & 0.03 & 0.09 & 0.12 & 121 \\
\hline $9890.70^{5}$ & & 8.40 & - & - & 1.200 & 1.270 & $-31.34^{h}$ & -4.42 & 0.06 & 0.12 & 0.16 & 70 \\
\hline
\end{tabular}

Column 2 contains the multiplet numbers accordingly to Moore (1972).

Column 13 contains solar equivalent widths.

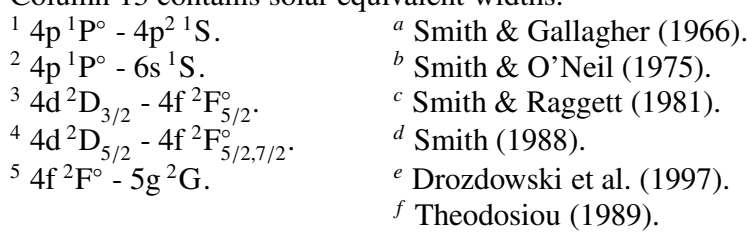

\author{
${ }^{g} A \& O^{\prime} M$. \\ ${ }^{h}$ Kurucz's calculations. \\ ${ }^{i}$ Smith (1981). \\ * from solar line profile fitting.
}

the model with $[\mathrm{Fe} / \mathrm{H}]=-1$. The maximum correction amounts to -0.03 dex.

Thus, the largest uncertainty of NLTE results for $\mathrm{Ca} \mathrm{I} / \mathrm{II}$ is caused by poor knowledge of collision processes. Below we empirically constrain collisional data by analyzing the $\mathrm{Ca}$ lines in solar (Sect. 4) and stellar (Sect. 6) spectra.

\section{Solar $\mathrm{Ca}$ abundance and $\mathrm{Ca} \mathrm{I} / \mathrm{Ca}$ II ionization equilibrium}

In this section, we derive the solar $\mathrm{Ca}$ abundance from the $\mathrm{Ca}$ I subordinate lines and the Ca II high excitation lines and examine the atomic data used in SE calculations and element abundance determinations. By taking advantage of the applied
NLTE approach, both weak and strong Ca lines are included in the analysis. An exception is the resonance lines in $\mathrm{Ca}$ I and $\mathrm{Ca}$ II and the $\mathrm{Ca}$ II lines of multiplet $3 \mathrm{~d}-4 \mathrm{p}$, because their cores and inner wings are influenced by the chromospheric temperature rise and by the non-thermal and depth-dependent chromospheric velocity field that is not part of the MAFAGS model of the solar atmosphere. However, here we check the wings of Ca I $\lambda 4226$ and Ca II $\lambda 8498$.

We used solar flux observations taken from the Kitt Peak Solar Atlas (Kurucz et al. 1984) and selected the Ca lines free of blends, the ones with only one distorted line wing (e.g., Ca I $\lambda 4425$ ), or those where the blending lines can be taken into account correctly (e.g., CaI $\lambda 6572$ in the wing of Balmer line $\mathrm{H} \alpha$ ). The investigated lines are listed in Table 4. 
Four different sets of oscillator strengths were applied and compared in this study. (i) The $f_{i j}$ values obtained from laboratory measurements are available for all selected $\mathrm{Ca}$ I lines and shown in Table 4 (column LAB), together with their sources; (ii) The $f_{i j}$ based on OP calculations are available for all optically permitted transitions in $\mathrm{Ca}$ I and $\mathrm{Ca}$ II, as are the data from (iii) the NIST; and (iv) VALD databases. We used the radiative widths obtained by Kurucz (1992) from radiative lifetimes which are accessible via the VALD database.

For $17 \mathrm{Ca}$ I lines, the $C_{6}$ values were computed from damping parameters given by Smith (1981) and based on the measured parameters for broadening by helium. For the remaining lines, we adopted $C_{6}$ values based on either $A \& O^{\prime} M$ or Kurucz calculations, giving a preference to the first source. The necessary data are accessible via the VALD database. The van der Waals damping parameters based on the perturbation theory of $A \& O^{\prime} M$ agree within 0.1 dex of $\log C_{6}$ with the quantum mechanic computations by Spielfiedel et al. (1991) for the Ca I multiplet $3\left(4 \mathrm{p}^{3} \mathrm{P}^{\circ}-5 \mathrm{~s}^{3} \mathrm{~S}\right)$ and by Kerkeni et al. (2004) for the CaI resonance line $\lambda 4226$. For four among five common Ca I multiplets, the predicted $A \& O^{\prime} M$ parameters lead to stronger collisional broadening compared to that from the experimental data of Smith (1981). The difference in $\log C_{6}$ ranges from 0.19 dex to 0.32 dex. The opposite is the case for the $\mathrm{Ca}$ I $\lambda 5261$ line with the experimental value of $\log C_{6}$ larger by 0.25 dex compared to the predicted one. For Ca I $\lambda 4425$ and the lines of Ca II multiplets $5 \mathrm{p}-5 \mathrm{~d}$ and $4 \mathrm{~d}-4 \mathrm{f}$, the $C_{6}$ values are obtained empirically from the fitting of solar line profiles. We note that not only $\lambda 4425$ but also two other lines of the Ca I multiplet $4 \mathrm{p}^{3} \mathrm{P}^{\circ}-4 \mathrm{~d}^{3} \mathrm{D}, \lambda 4454$ and $\lambda 4455$, though being blended, certainly cannot be fitted with $\log C_{6}=-30.23$ as computed from $A \& O^{\prime} M$ data. We find $\log C_{6}=-30.9$ for these lines. The best fit of $\lambda 4425$ is shown in Fig. 5. For the Ca II multiplet $5 \mathrm{p}-5 \mathrm{~d}, \log C_{6}=-30.85$ is found from the requirement that element abundances derived from the weaker line, $\lambda 8254\left(W_{\lambda}=18 \mathrm{~m} \AA\right)$, and the stronger line, $\lambda 8248\left(W_{\lambda}=\right.$ $65 \mathrm{~mA}$ ), must be equal. In contrast to multiplet $5 \mathrm{p}-5 \mathrm{~d}$, the lines of the Ca II multiplet $4 \mathrm{~d}-4 \mathrm{f}, \lambda 8912$ and $\lambda 8927$, are both sensitive to a variation in the van der Waals damping constant. We assumed that the broadening parameter calculated by Kurucz is underestimated to the same extent as the corresponding value for multiplet $5 \mathrm{p}-5 \mathrm{~d}$ and, thus, obtained $\log C_{6}=-31.1$ for multiplet $4 \mathrm{~d}-4 \mathrm{f}$. The best fit of $\lambda 8927$ is shown in Fig. 5. The atomic data we used are presented in Table 4.

A depth-independent microturbulence of $0.9 \mathrm{~km} \mathrm{~s}^{-1}$ is adopted. Our synthetic flux profiles are convolved with a profile that combines a rotational broadening of $1.8 \mathrm{~km} \mathrm{~s}^{-1}$ and broadening by macroturbulence with a radial-tangential profile of $V_{\text {mac }}=3 \mathrm{~km} \mathrm{~s}^{-1}$ to $V_{\text {mac }}=4 \mathrm{~km} \mathrm{~s}^{-1}$ for different lines.

For each investigated line, the product $\log g f \varepsilon_{\mathrm{Ca}}^{\odot}$ was obtained from solar line-profile fitting under various line-formation assumptions: NLTE $S_{\mathrm{H}}=0, S_{\mathrm{H}}=0.1$, and $S_{\mathrm{H}}=1$ and LTE. Table 4 presents the NLTE values $\log g f \varepsilon_{\mathrm{Ca}}^{\odot}$ derived assuming $S_{\mathrm{H}}=0$ and abundance corrections $\Delta_{\mathrm{X}}=\log \varepsilon_{\mathrm{X}}-\log \varepsilon_{\mathrm{S}_{\mathrm{H}}=0}$ where $\mathrm{X}$ takes the meaning of $0.1,1$, and LTE for $S_{\mathrm{H}}=0.1$, $S_{\mathrm{H}}=1$, and the LTE assumption, respectively. By definition, $\Delta_{\text {LTE }}$ (Table 4$)=-\Delta_{\text {NLTE }}$ (Table 2). It is interesting to note that, for every investigated line, the $\Delta_{\text {NLTE }}$ given in the first string of Table 2 does not coincide in absolute value with $\Delta_{\text {LTE }}$ in Table 4. This can be understood because the NLTE corrections in Table 2 were calculated from comparison of NLTE and LTE equivalent widths, while abundance corrections in Table 4 are based on the analysis of line profiles. For each spectral line, the best NLTE fit, independent of its $S_{\mathrm{H}}$ value, reproduces the line core better than
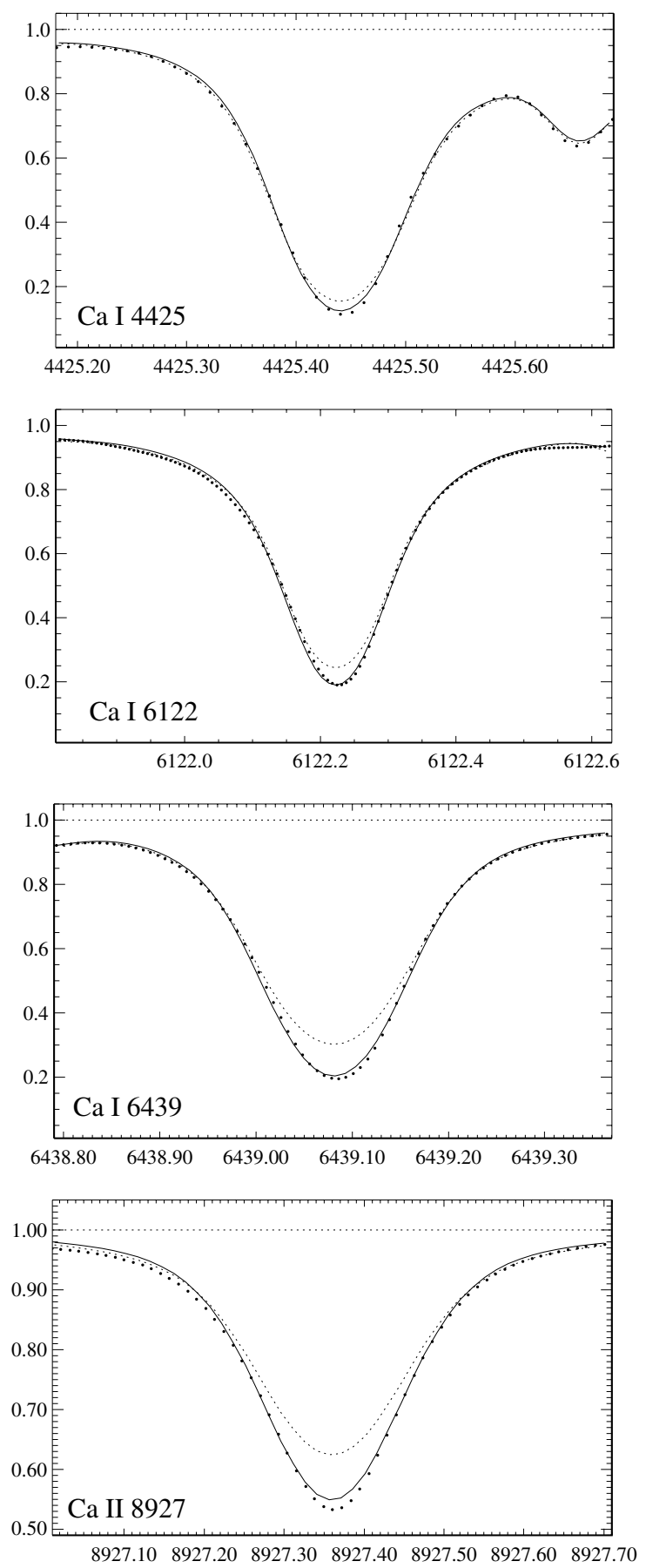

Fig. 5. Synthetic NLTE $\left(S_{\mathrm{H}}=0\right.$, continuous line) and LTE (dotted line) flux profiles of the Ca I $\lambda 4425, \lambda 6122$, and $\lambda 6439$ and Ca II $\lambda 8927$ lines compared with the observed spectrum of the Kurucz et al. (1984) solar flux atlas (bold dots). The fitting parameters are presented in Table 4. It should be stressed that different $\mathrm{Ca}$ abundances are required to achieve the best fits of the observed profile in the $\operatorname{NLTE}\left(S_{\mathrm{H}}=0\right)$ and LTE cases. Everywhere $V_{\text {mic }}=0.9 \mathrm{~km} \mathrm{~s}^{-1}$.

the LTE one. However, for many lines, even the NLTE fit is not perfect. As an example, we show the best NLTE $\left(S_{\mathrm{H}}=0\right)$ and LTE fits in Fig. 5 for three lines of CaI and $\lambda 8927$ of Ca II. It is clearly seen that the observed line core of Ca I $\lambda 6439$ is asymmetric. This is, most probably, due to atmospheric inhomogeneity and, therefore, cannot be reproduced in the framework of a $1 \mathrm{D}$ analysis. 

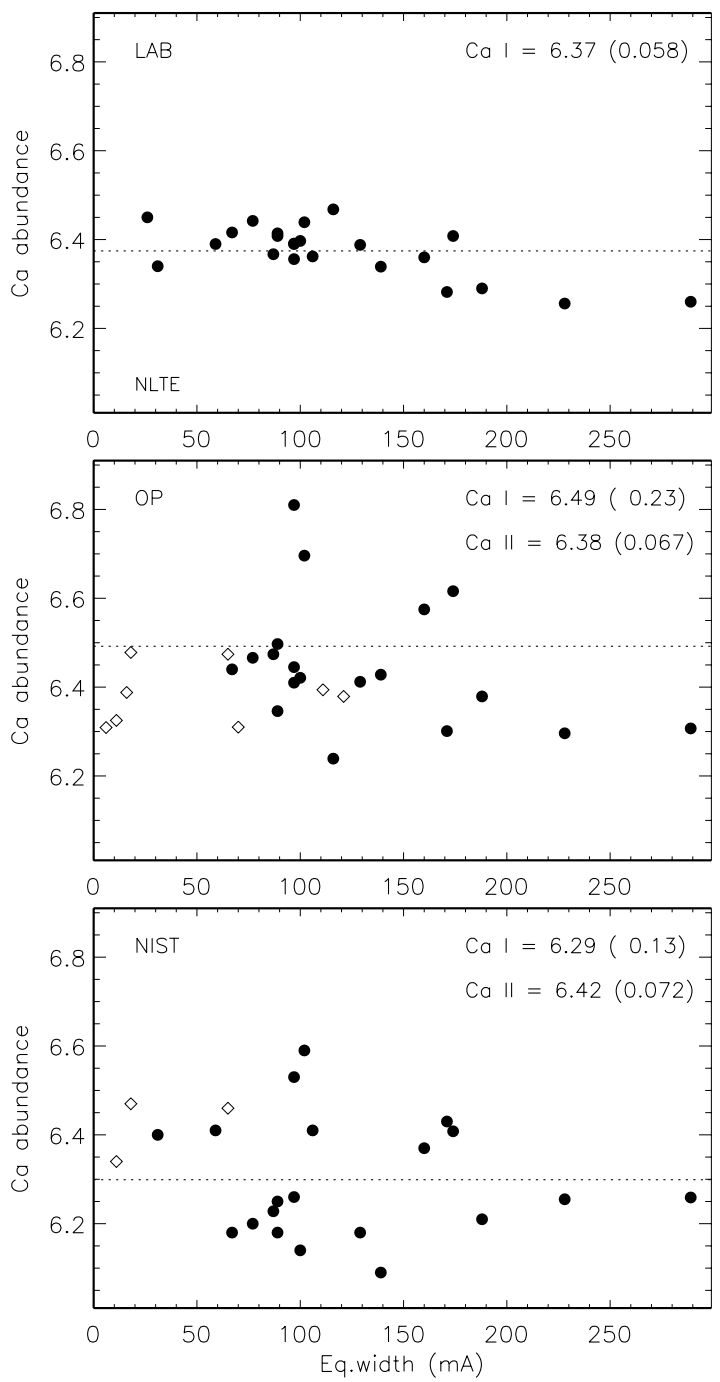

Fig. 6. Solar NLTE $\left(S_{\mathrm{H}}=0\right)$ Ca abundances from the Ca I (filled circles) and Ca II (open diamonds) lines plotted as a function of the line equivalent width. Top, middle, and bottom panels show results based on the measured $f_{i j}$, OP, and NIST data, respectively. In each panel, the mean abundance derived from the $\mathrm{Ca}$ I lines is shown by the dotted line and the mean values and their standard deviations are quoted for $\mathrm{Ca}$ I and Ca II.

Using the obtained values $\log g f \varepsilon_{\mathrm{Ca}}^{\odot}$ and $f_{i j}$ from different sets, we computed $\mathrm{Ca}$ abundances from individual lines. Figure 6 illustrates results for NLTE $\left(S_{\mathrm{H}}=0\right)$ calculations. It can be seen that the laboratory oscillator strengths provide the highest accuracy in the absolute solar abundance derived from the lines of CaI. The NLTE and LTE averages from 23 lines are $\log \varepsilon_{\mathrm{CaI}}\left(\mathrm{LAB}, S_{\mathrm{H}}=0\right)=6.37 \pm 0.06, \log \varepsilon_{\mathrm{CaI}}\left(\mathrm{LAB}, S_{\mathrm{H}}=\right.$ $0.1)=6.36 \pm 0.06, \log \varepsilon_{\mathrm{CaI}}\left(\mathrm{LAB}, S_{\mathrm{H}}=1\right)=6.35 \pm 0.06$, and $\log \varepsilon_{\mathrm{CaI}}(\mathrm{LAB}, \mathrm{LTE})=6.34 \pm 0.06$. Throughout this paper, the standard deviations are quoted. The NLTE effects for Ca I in the Sun are very small, and we present the results only for $S_{\mathrm{H}}=$ 0 . The $\mathrm{Ca}$ abundances based on $\mathrm{OP} f_{i j}$ for $20 \mathrm{Ca}$ I lines reveal a large spread of data with the mean values $\log \varepsilon_{\mathrm{CaI}}\left(\mathrm{OP}, S_{\mathrm{H}}=\right.$ $0)=6.49 \pm 0.23$ and $\log \varepsilon_{\mathrm{CaI}}(\mathrm{OP}, \mathrm{LTE})=6.46 \pm 0.21$. Excluding the one line, $\lambda 5867$, with the largest contribution to standard deviation, we obtain $\log \varepsilon_{\mathrm{CaI}}\left(\mathrm{OP}, S_{\mathrm{H}}=0\right)=6.45 \pm 0.14$ and $\log \varepsilon_{\mathrm{CaI}}(\mathrm{OP}, \mathrm{LTE})=6.43 \pm 0.14$. From $20 \mathrm{Ca}$ I lines with the NIST data available, the averages equal $\log \varepsilon_{\mathrm{CaI}}\left(\mathrm{NIST}, S_{\mathrm{H}}=0\right)=$ $6.29 \pm 0.13$ and $\log \varepsilon_{\mathrm{CaI}}(\mathrm{NIST}, \mathrm{LTE})=6.26 \pm 0.13$. The results based on the VALD data are close to those for laboratory measurements because, for most lines investigated, the VALD's oscillator strengths were taken from Smith \& Raggett (1981). Tests show that the NLTE abundance from $\mathrm{Ca}$ I lines is not sensitive to a variation in electronic collisional rates in SE calculations. The use of the formula of van Regemorter (1962) and the IPM data (Seaton 1962a) leads to the mean $\mathrm{Ca}$ abundances, consistent within 0.01 dex. In either case, either NLTE or LTE, for any set of $f_{i j}$, no significant correlation of individual $\mathrm{Ca}$ abundance is found with the line strength.

Only predicted oscillator strengths are available for the Ca II lines that were used to determine the absolute solar abundance from $\mathrm{Ca}$ II lines. OP data provide high accuracy for the desired value provided that an SE approach is applied. NLTE corrections remove a trend of the CaII abundance with a line strength obtained under the LTE assumption. Averages from eight lines are $\log \varepsilon_{\text {CaII }}\left(\mathrm{OP}, S_{\mathrm{H}}=0\right)=6.38 \pm 0.07, \log \varepsilon_{\mathrm{CaII}}(\mathrm{OP}$, $\left.S_{\mathrm{H}}=0.1\right)=6.40 \pm 0.06$, and $\log \varepsilon_{\mathrm{CaII}}\left(\mathrm{OP}, S_{\mathrm{H}}=1\right)=6.43 \pm 0.08$. It should be noted that a variation in $\log C_{6}$ values within $0.6 \mathrm{dex}$ for the lines of the multiplets $5 p-5 d$ and $4 d-4$ f sensitive to van der Waals broadening changes the mean Ca II abundance by only 0.01 dex. The NIST database contains only three Ca II lines of interest, and their $f_{i j}$ are very close to the corresponding values from OP calculations. For the VALD data, the NLTE and LTE mean Ca II abundances equal $\log \varepsilon_{\text {CaII }}\left(\right.$ VALD, $\left.S_{\mathrm{H}}=0\right)=$ $6.65 \pm 0.32$ and $\log \varepsilon_{\text {CaII }}($ VALD, LTE $)=6.74 \pm 0.29$. The large standard deviation is mainly caused by $\lambda 6456$ and $\lambda 5339$. In contrast to Ca I, the NLTE Ca II abundance is sensitive to a variation in electronic collisional rates in SE calculations. The value determined using the formula of van Regemorter (1962) and assuming $S_{\mathrm{H}}=0$ is higher by 0.05 dex compared to the corresponding value obtained for the standard recipe of electronic collisional rates.

Taking the highest accuracy of the mean values into account, we preferred to use as final values the $\mathrm{Ca}$ II abundance based on OP oscillator strengths and $\mathrm{Ca} I$ abundance based on the laboratory $f_{i j}$. They agree within $\leq 0.04$ dex provided that $S_{\mathrm{H}} \leq 0.1$ and the standard recipe of electronic collisional rates is applied. In any other case, the difference equals $0.08 \mathrm{dex}\left(S_{\mathrm{H}}=1\right.$ or van Regemorter's electronic collisional rates) to 0.12 dex (LTE). Thus, using the theoretical MAFAGS model atmosphere, we find the solar $\mathrm{Ca}$ abundance to lie between 6.36 and 6.40 .

We also obtain here the fitting parameters of solar Ca I $\lambda 4226$ and Ca II $\lambda 8498$ lines necessary for further analysis of metal-poor stars. For both lines, oscillator strengths are taken from laboratory measurements (Smith \& Gallagher 1966 for $\lambda$ 4226; Theodosiou 1989 for $\lambda$ 8498), and the van der Waals damping constants are based on $A \& O^{\prime} M$ 's data (Table 4). For $\lambda 8498$, the isotope structure is taken into account with atomic data from Table 1. A good fit of the observed solar flux profile of $\lambda 4226$ in the wings is achieved for $\log \varepsilon_{\mathrm{Ca}}=$ 6.29 when hydrogenic collisions are ignored and for $\log \varepsilon_{\mathrm{Ca}}=$ 6.21 in the LTE case. The NLTE effects are negligible for the $\lambda 8498$ line wings. The best fit is achieved at a Ca abundance of $\log \varepsilon_{\mathrm{Ca}}=6.28$.

Recent determinations of solar photospheric $\mathrm{Ca}$ abundance based on a 3D LTE analysis (Asplund et al. 2005) give "excellent agreement between the two ionization stages" and the average of the two: $\log \varepsilon_{\mathrm{Ca}}=6.31 \pm 0.04$. The average obtained from our 1D NLTE analysis is $\log \varepsilon_{\mathrm{Ca}}=6.38 \pm 0.06$. We showed above that the absolute $\mathrm{Ca}$ abundance depends on the adopted values of oscillator strengths and van der Waals damping constants. The cited paper of Asplund et al. does not give enough information for a discussion of the possible sources of the found discrepancy. 
Table 5. Stellar parameters and obtained NLTE elemental abundance ratios of the selected stars.

\begin{tabular}{rccccccc}
\hline \hline $\mathrm{HD} / \mathrm{BD}$ & $T_{\text {eff }}$ & $\log g$ & {$[\mathrm{Fe} / \mathrm{H}]_{\mathrm{II}}$} & $V_{\mathrm{mic}}\left(\mathrm{km} \mathrm{s}^{-1}\right)$ & {$[\mathrm{Mg} / \mathrm{Fe}]_{\mathrm{I}}$} & \multicolumn{1}{c}{$[\mathrm{Ca} / \mathrm{Fe}]_{\mathrm{I}}$} & \multicolumn{1}{c}{$[\mathrm{Ca} / \mathrm{Fe}]_{\mathrm{II}}$} \\
\hline 19445 & 6030 & $4.40 \pm 0.06$ & $-2.08 \pm 0.05$ & $1.6 \pm 0.2$ & $0.49 \pm 0.03$ & $0.36 \pm 0.04$ & 0.40 \\
29907 & 5500 & $4.64 \pm 0.06$ & $-1.60 \pm 0.02$ & $0.7 \pm 0.1$ & $0.35 \pm 0.01$ & $0.33 \pm 0.05$ & \\
59392 & 6010 & $4.02 \pm 0.15$ & $-1.60 \pm 0.03$ & $1.4 \pm 0.2$ & $0.33 \pm 0.05$ & $0.26 \pm 0.04$ & \\
61421 & 6510 & $3.96 \pm 0.02$ & $-0.03 \pm 0.04$ & $1.8 \pm 0.1$ & $0.00 \pm 0.06$ & $-0.11 \pm 0.03$ & $0.10 \pm 0.05$ \\
84937 & 6350 & $4.00 \pm 0.12$ & $-2.16 \pm 0.05$ & $1.8 \pm 0.2$ & $0.39 \pm 0.04$ & $0.43 \pm 0.04$ & 0.38 \\
103095 & 5070 & $4.69 \pm 0.06$ & $-1.35 \pm 0.03$ & $0.8 \pm 0.1$ & $0.25 \pm 0.03$ & $0.30 \pm 0.04$ & 0.29 \\
140283 & 5810 & $3.68 \pm 0.06$ & $-2.43 \pm 0.05$ & $1.6 \pm 0.2$ & $0.32 \pm 0.02$ & $0.29 \pm 0.06$ & 0.24 \\
$-4^{\circ} 3208$ & 6280 & $4.08 \pm 0.24$ & $-2.30 \pm 0.06$ & $1.8 \pm 0.2$ & $0.41 \pm 0.01$ & $0.43 \pm 0.04$ & 0.50 \\
\hline
\end{tabular}

\section{Stellar sample, observations, and stellar parameters}

Our sample consists of eight stars. HD 61421 (Procyon) is selected as a fundamental star with nearly the same list of detected $\mathrm{Ca}$ lines as in the Sun. It is especially important for checking the $\mathrm{Ca}$ II high excitation lines. The other objects are metal-poor stars with metallicity ranging between $[\mathrm{Fe} / \mathrm{H}]=-1.35$ and $[\mathrm{Fe} / \mathrm{H}]=$ -2.43 . They give an opportunity to study a formation of some of the strongest $\mathrm{Ca}$ lines, $\mathrm{Ca}$ I $\lambda 4226$ and $\mathrm{Ca}$ II $\lambda 8498$, which presumably have a purely photospheric origin in this metallicity range.

Four metal-poor stars, HD 29907, HD 59392, HD 140283, and $\mathrm{BD}-4^{\circ} 3208$, were observed using the Ultraviolet and Visual Echelle Spectrograph UVES at the $8 \mathrm{~m}$ ESO VLT UT2 telescope on Cerro Paranal. At least two exposures were obtained for each star. The spectral resolving power is about 60000 . The data cover an approximate spectral range of 4000-7000 A. The signal-to-noise ratio is 200 or higher over the whole spectral range. No near-IR spectra for HD 29907 and HD 59392 are available to us.

Observational data for all other stars (and for Ca II 8498 in $\left.\mathrm{BD}-4^{\circ} 3208\right)$ were taken from Korn et al. (2003) with overall similar data-quality specifications as for the UVES data. For these data, the wavelength coverage is 4200-9000 А. Highquality observations for Ca II 8498 in HD 140283 were taken from the ESO UVESPOP survey (Bagnulo et al. 2005).

We used stellar parameters determined in our earlier studies (Korn et al. 2003; Mashonkina et al. 2003). In short, effective temperatures were determined from Balmer line-profile fitting, $\mathrm{H} \alpha$ and $\mathrm{H} \beta$, with a statistical error estimated at the level of $70 \mathrm{~K}$, surface gravities from the HIPPARCos parallaxes with masses determined from the tracks of VandenBerg et al. (2000). The errors obtained from adding the squared errors of parallax and mass are quoted in Table 5. The iron abundance and microturbulence velocity were obtained requiring the derived $[\mathrm{Fe} / \mathrm{H}]_{\mathrm{II}}^{2}$ abundances not to depend on line strength. For metalpoor stars, $\alpha$-enhanced models were calculated with the abundances of $\alpha$-elements $\mathrm{O}, \mathrm{Mg}, \mathrm{Si}$, and $\mathrm{Ca}$, scaled by the stellar $\mathrm{Mg} / \mathrm{Fe}$ ratio. The latter was determined in this study from NLTE analysis of four Mg I lines, $\lambda 4571, \lambda 4703, \lambda 5528$, and $\lambda 5711$. We used the model atom and atomic data for $\mathrm{Mg} \mathrm{I}$ described by Gehren et al. (2004). Stellar parameters are given in Table 5 .

The microturbulence values are somewhat lower than values previously published by us, since we now utilize the broadening parameters of Barklem \& Aspelund-Johansson (2005), which play a role even in the metal-poor stars as the analysis is

2 The subscript in $[\mathrm{X} / \mathrm{Y}]_{\mathrm{II}}$ indicates that the abundance for element $\mathrm{X}$ is determined from the singly ionized species. Likewise, a subscript I refers to neutral lines used in the abundance determination. differential to the Sun. In all metal-poor stars, distinguishing of the microturbulence values relies heavily on the three strong Fe II lines of multiplet 42 ( $\lambda \lambda 4923,5018$, and $5169 \AA)$. All three lines seem to require substantially higher microturbulence values in the Sun. Enforcing a fixed solar microturbulence of $V_{\text {mic }}=0.9 \mathrm{~km} \mathrm{~s}^{-1}$ thus means that they cannot be well-fitted. We estimate that, depending on the choices made in the fitting of solar lines and the line selection for the metal-poor stars, microturbulence values can easily vary by $0.2 \mathrm{~km} \mathrm{~s}^{-1}$. In particular, lower values seem possible (e.g. disregarding Fe II $5169 \AA$ A). This is important to bear in mind for the discussion of the strong lines in Sects. 6 and 7.

Our results are based on line profile analysis. In order to compare with observations, computed synthetic profiles are convolved with a profile that combines instrumental broadening with a Gaussian profile and broadening by macroturbulence with a radial-tangential profile. Only slow rotators are included in our sample, $V \sin i \leq 1.5 \mathrm{~km} \mathrm{~s}^{-1}$, except for Procyon with $V \sin i=2.6 \mathrm{~km} \mathrm{~s}^{-1}$ (Fuhrmann 1998) and therefore the rotational velocity and macroturbulence value cannot be separated at the spectral resolving power of our spectra. We thus treat their overall effect as radial-tangential macroturbulence. Rotational broadening and broadening by macroturbulence are treated separately only for Procyon. The macroturbulence value was determined for each star in our previous studies from the analysis of an extended list of lines of $\mathrm{Fe} \mathrm{I} / \mathrm{II}, \mathrm{MgI}$, etc. Here, $V_{\operatorname{mac}}$ was allowed to vary by $\pm 0.3 \mathrm{~km} \mathrm{~s}^{-1}(1 \sigma)$.

\section{The $\mathrm{Ca}$ I versus $\mathrm{Ca}$ II in the selected stars}

In this section, we test NLTE formation of the CaII lines in two steps. In the first one, the Ca II high-excitation lines are examined in the solar metallicity star Procyon. These lines are more sensitive to details of NLTE calculations than $\lambda 8498$ (see Table 3 ). We then derive element abundances from the CaI lines and from Ca II $\lambda 8498$ for five metalpoor stars and inspect the difference $\Delta \log \varepsilon(\mathrm{Ca}$ I $-\mathrm{Ca}$ II $)$. In the temperature regime we are concerned with, and at metallicities $[\mathrm{Fe} / \mathrm{H}]$ between -2 and -3 , the equivalent width ratio $W(\mathrm{Ca} \mathrm{I}) / W(\mathrm{Ca}$ II 8498$)$ is weakly sensitive to a variation in $T_{\text {eff }}$ and $\log g$, independent of what Ca I line is taken. For example, $\log W(\mathrm{Ca}$ I 6439) $/ W(\mathrm{Ca}$ II 8498) $=-0.88,-0.81,-0.77$, and -0.78 for the models with $T_{\text {eff }} / \log g /[\mathrm{Fe} / \mathrm{H}]=5000 \mathrm{~K} / 3.0 /$ $-2,5000 \mathrm{~K} / 4.0 /-2,6000 \mathrm{~K} / 3.0 /-2$, and $6000 \mathrm{~K} / 4.0 /-2$, respectively. This can be understood because the van der Waals broadened line wings significantly contribute to $W(\mathrm{Ca}$ II 8498$)$, even at $[\mathrm{Ca} / \mathrm{H}]=-2.6$ (for the models with $[\mathrm{Fe} / \mathrm{H}]=-3$ ). When $\log g$ increases, strengthening of the $\lambda 8498$ line wings is compensated for by weakening NLTE effects. In such conditions, a difference between $\mathrm{Ca}$ abundances determined from two ionization stages, if present, will point to shortcomings in 


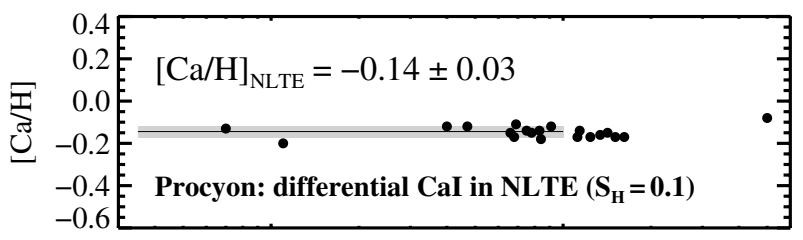

$10 \quad 100$

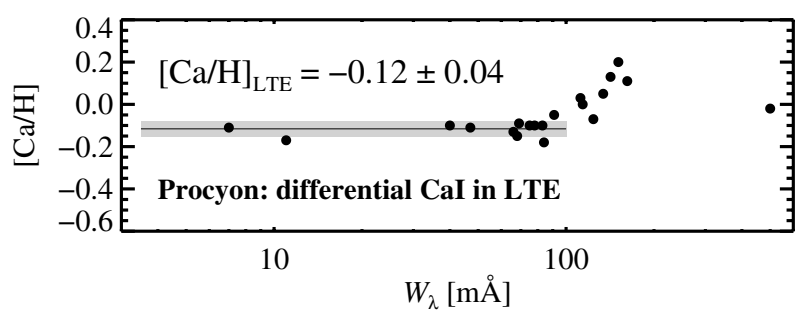

Fig. 7. Trends in abundance with line strength determined from the $\mathrm{Ca}$ I lines in Procyon for our best NLTE model (top) and under the assumption of LTE (bottom). Note the steep trend of the LTE $[\mathrm{Ca} / \mathrm{H}]$ values with line strength above $W_{\lambda}=100 \mathrm{~m} \AA$. The mean values given are computed only from weak lines $\left(W_{\lambda} \leq 100 \mathrm{~m} \AA\right)$.

the NLTE treatment of Ca lines rather than to the uncertainty of stellar parameters.

Analysis of stellar spectra is made line-by-line differentially with respect to the Sun. At the LTE assumption, the cores of many lines cannot be fitted. In such cases, the $\mathrm{Ca}$ abundance is derived from the line wing fitting.

Procyon

Irrespective of the assumed efficiency of hydrogen collisions, average NLTE effects are very small among weak Ca I lines $\left(W_{\lambda} \leq\right.$ $100 \mathrm{m \AA})$ and vary between $-0.02 \operatorname{dex}\left(S_{\mathrm{H}}=1\right)$ and $-0.03 \mathrm{dex}$ $\left(S_{\mathrm{H}}=0\right)$. However, the $\mathrm{SE}$ approach is able to remove a steep trend with line strength among strong $\mathrm{Ca}$ I lines seen in LTE (see Fig. 7). For individual strong lines (like Ca I $6439 \AA$ A), NLTE corrections exceed -0.3 dex. Among weak lines $\left(W_{\lambda}<100 \mathrm{~m} \AA\right.$ ), the line-to-line scatter is reduced by all three NLTE models and attains its minimal value $(\sigma=0.027 \mathrm{dex})$ at $S_{\mathrm{H}}=0.1$. Surprisingly, the mean abundance derived from $\mathrm{Ca}$ I lines is clearly subsolar, $[\mathrm{Ca} / \mathrm{H}]_{\mathrm{I}}=-0.12 \pm 0.04$ (LTE) to $-0.15 \pm 0.04$ $\left(S_{\mathrm{H}}=0\right)$.

Abundances for $\mathrm{Ca}$ II are derived from six high-excitation lines. The mean NLTE value is $[\mathrm{Ca} / \mathrm{H}]_{\mathrm{II}}(\mathrm{NLTE})=0.07 \pm 0.05$, independent of the assumed strength of hydrogenic collisions. The LTE abundances show a mean value of $[\mathrm{Ca} / \mathrm{H}]_{\mathrm{II}}(\mathrm{LTE})=$ $0.14 \pm 0.05$. Applying the formula of van Regemorter (1962) to a calculation of electronic collisional rates results in an even larger difference $\Delta \log \varepsilon(\mathrm{Ca}$ I $-\mathrm{Ca}$ II $)$.

Can the stellar parameters cause this discrepancy? Allende Prieto et al. (2002) estimated an error of $49 \mathrm{~K}$ for the effective temperature. Ramirez \& Melendez (2005) revised the fundamental effective temperature of Procyon to $T_{\text {eff }}^{\text {fund }}=6532 \pm 39 \mathrm{~K}$ and derived $T_{\text {eff }}^{\mathrm{IRFM}}=6591 \pm 73 \mathrm{~K}$ using their infrared flux method. Assuming $T_{\text {eff }}=6590 \mathrm{~K}$ (a value favored by Korn et al. 2003 for the NLTE ionization equilibrium of $\mathrm{Fe} \mathrm{I} / \mathrm{II})$ and keeping the values of $\log g,[\mathrm{Fe} / \mathrm{H}]$, and $V_{\text {mic }}$ unchanged, we obtain mean values $[\mathrm{Ca} / \mathrm{H}]_{\mathrm{I}}(\mathrm{NLTE})=-0.10 \pm$ 0.04 and $[\mathrm{Ca} / \mathrm{H}]_{\mathrm{II}}(\mathrm{NLTE})=0.05 \pm 0.05$. As the discrepancy lies with weak lines, modifications to the microturbulence value cannot remove this discrepancy either. Aufdenberg et al. (2005) conclude that the interferometric data provide evidence of convective overshooting in Procyon's atmosphere. We checked a model that includes an "approximate overshooting"

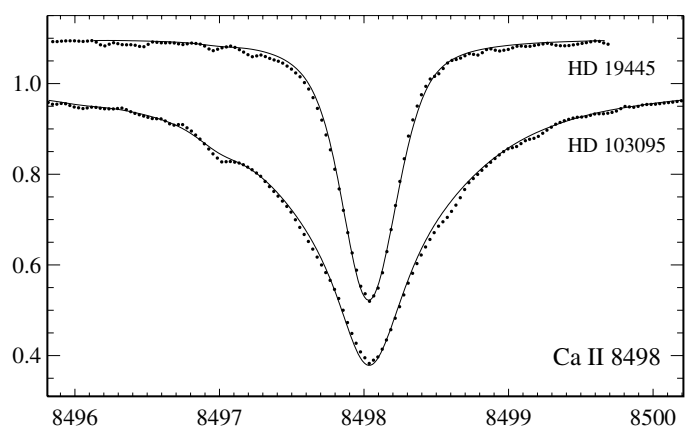

Fig. 8. The best NLTE $\left(S_{\mathrm{H}}=0.1\right)$ fits (continuous line) of the observed profiles of Ca II $\lambda 8498$ in spectra of HD 19445 and HD 103095 (bold dots). The obtained NLTE Ca abundances are presented in Table 5. For HD 19445, line profiles are shifted along $Y$ axis.

prescription for convective flux transport in a mixing-length formalism according to Castelli et al. (1997). The investigated lines of both $\mathrm{CaI}$ and $\mathrm{Ca}$ II become weaker in this model compared to our standard MAFAGS model, and the derived abundances of $\mathrm{Ca}$ I and Ca II increase by $0.08-0.10 \mathrm{dex}$ for different lines. However, the difference $\Delta \log \varepsilon(\mathrm{Ca}$ I $-\mathrm{Ca}$ II $)$ remains at the same level.

The effect of atmospheric temperature inhomogeneities on $\mathrm{Ca}$ I/II is expected to be within the same order of magnitude as that for neutral and singly-ionized iron lines, as calculated by Allende Prieto et al. (2002). They show that weak lines $\left(W_{\lambda} \leq 50 \mathrm{~m} \AA\right)$ of both ionization stages, Fe I and Fe II, are weakened compared to a classical 1D analysis, such that the derived $\mathrm{Fe}$ abundance increases by 0.05 dex and 0.04 dex, respectively. Three-dimensional simulations might lead to the higher $\mathrm{Ca}$ abundance for Procyon compared to our results; however, the discrepancy between CaI and Ca II is unlikely to be removed. Thus, while our NLTE analysis leads to a better agreement of the $\mathrm{Ca}$ abundance from the two ionization stages, the discrepancy exceeds $3 \sigma$ under standard assumptions about Procyon's stellar parameters.

\section{HD 19445}

The abundance is determined from $15 \mathrm{Ca}$ I lines with equivalent widths between $4 \mathrm{~m} \AA$ and $63 \mathrm{~m} \AA$. The accuracy of the obtained differential abundances is at the level of $\sigma=0.04$ dex for $S_{\mathrm{H}}=0$, 0.1 and the LTE case and slightly worse, $\sigma=0.06 \mathrm{dex}$, for $S_{\mathrm{H}}=$ 1. The difference between NLTE and LTE Ca I abundances depends strongly on the assumed value of $S_{\mathrm{H}}$. It equals $+0.13 \mathrm{dex}$ for $S_{\mathrm{H}}=0$ and reduces down to +0.03 dex for $S_{\mathrm{H}}=1$. In contrast, NLTE abundances from Ca II $\lambda 8498$ are lower than in LTE. The best fit of $\lambda 8498$ achieved at $S_{\mathrm{H}}=0.1$ and $[\mathrm{Ca} / \mathrm{Fe}]=0.40$ is shown in Fig. 8. The difference between $\mathrm{Ca}$ I and $\mathrm{Ca}$ II abundances is found to be $0.04 \mathrm{dex},-0.04 \mathrm{dex},-0.11 \mathrm{dex}$, and -0.19 dex for $S_{\mathrm{H}}=0, S_{\mathrm{H}}=0.1, S_{\mathrm{H}}=1$, and LTE, respectively. An uncertainty in microturbulence of $0.2 \mathrm{~km} \mathrm{~s}^{-1}$ hardly affects the CaI abundance and leads to a change in Ca II of 0.03 dex. This does not destroy the agreement of the NLTE Ca I and Ca II abundances if $S_{\mathrm{H}}=0$ or 0.1 is assumed.

HD 84937

The abundance obtained from $\mathrm{Ca}$ I lines varies between $[\mathrm{Ca} / \mathrm{Fe}]_{\mathrm{I}}=0.47 \pm 0.04$ and $[\mathrm{Ca} / \mathrm{Fe}]_{\mathrm{I}}=0.39 \pm 0.04$, when changing $S_{\mathrm{H}}$ between 0 and $\infty$ (LTE). Similar to HD 19445, the abundances from the two ionization stages agree much more closely in the NLTE case than in LTE one $-\Delta \log \varepsilon(\mathrm{Ca} \mathrm{I}-\mathrm{Ca}$ II $)=$ +0.11 dex and -0.02 dex for NLTE abundances at $S_{\mathrm{H}}=0$ and 1 , respectively - while the difference of LTE abundances equals 
-0.23 dex. The best agreement is found for an $S_{\mathrm{H}}$ value between 0.1 and 1 .

\section{HD 103095}

The determined NLTE abundances range between $[\mathrm{Ca} / \mathrm{Fe}]_{\mathrm{I}}=$ $0.37 \pm 0.04$ and $[\mathrm{Ca} / \mathrm{Fe}]_{\mathrm{I}}=0.29 \pm 0.04$, depending on the assumed $S_{\mathrm{H}}$ value. NLTE effects for Ca II $\lambda 8498$ occur only in the very core, and the same $\mathrm{Ca}$ abundance is obtained with $[\mathrm{Ca} / \mathrm{Fe}]_{\mathrm{II}}=0.29$, independent of the theory of line formation used. The best fit of this line is achieved for $S_{\mathrm{H}}=0.1$ (Fig. 8). The difference between $\mathrm{Ca}$ I and $\mathrm{Ca}$ II is within the mutual error bars in all cases: $\Delta \log \varepsilon(\mathrm{Ca} \mathrm{I}-\mathrm{Ca}$ II $)=+0.08 \mathrm{dex},+0.01 \mathrm{dex}$, $0.00 \mathrm{dex}$, and +0.03 dex for $S_{\mathrm{H}}=0,0.1,1$, and LTE, respectively. It should be noted that the discrepancy is larger when hydrogenic collisions are neglected (cf. HD 84937 above).

HD 140283

The $[\mathrm{Ca} / \mathrm{Fe}]_{\mathrm{I}}$ ratios vary between $+0.16 \pm 0.03$ (LTE) and $+0.33 \pm 0.03\left(S_{\mathrm{H}}=0\right)$ and are determined from six lines with equivalent widths between 6 and $40 \mathrm{~m} \AA$. At a microturbulence value of $V_{\text {mic }}=1.65 \mathrm{~km} \mathrm{~s}^{-1}$, the CaI resonance line $\left(W_{\lambda}=\right.$ $146 \mathrm{~m} \AA$ ) is in good agreement with the weak lines under the assumption of LTE, but yields abundances that are too low for all NLTE models. We comment on this behavior in the next section. As in the case of HD 84937, $\Delta \log \varepsilon(\mathrm{Ca}$ I $-\mathrm{Ca}$ II $)$ vanishes between $S_{\mathrm{H}}=0.1$ and 1 . In LTE, however, its value is -0.3 dex. BD $-4^{\circ} 3208$

This star is found to be very similar to HD 84937, both with respect to its stellar parameters and its behavior in terms of $\Delta \log \varepsilon(\mathrm{Ca} \mathrm{I}-\mathrm{Ca}$ II $)$. The abundances obtained from $14 \mathrm{Ca}$ I lines with equivalent widths between $4 \mathrm{~m} \AA$ and $40 \mathrm{~m} \AA$ give mean values of $[\mathrm{Ca} / \mathrm{Fe}]_{\mathrm{I}}\left(S_{\mathrm{H}}=0.1\right)=0.43 \pm 0.04$ and $[\mathrm{Ca} / \mathrm{Fe}]_{\mathrm{I}}(\mathrm{LTE})=$ $0.34 \pm 0.04$. Good agreement between $\mathrm{Ca} \mathrm{I}$ and $\mathrm{Ca}$ II is again found for an $S_{\mathrm{H}}$ range of 0 to 0.1 , while the difference increases above $S_{\mathrm{H}}=0.1$ and reaches $-0.35 \mathrm{dex}$ in the case of LTE.

Summarizing our results for the five metal-poor stars, we conclude that, within the modelling uncertainties, NLTE leads to consistent $\mathrm{Ca}$ abundances derived from the two ionization stages, while LTE fails to give consistent results. We find that ignoring hydrogenic collisions results in NLTE effects that are too strong for Ca I/II. Based on the results obtained for the Sun and the stars presented in this section, our best choice for a scaling factor applied to Steenbock \& Holweger's (1984) formula is $S_{\mathrm{H}}=0.1$. Final Ca I and Ca II abundances corresponding to $S_{\mathrm{H}}=$ 0.1 are presented in Table 5 .

\section{The CaI resonance line versus subordinate lines in metal-poor stars}

Ca I $\lambda 4226$ is the only neutral calcium line that can be detected in extremely metal-poor stars. In this section, we test the NLTE formation of this line using the metal-poor stars where, on one hand, the resonance line has a probably purely photospheric origin and, on other hand, Ca I subordinate lines are still measurable and provide a reliable value for the $\mathrm{Ca}$ abundance. In addition to the five metal-poor stars discussed above, another two stars are studied here. We start by determining the $[\mathrm{Ca} / \mathrm{Fe}]_{\mathrm{I}}$ ratio for them.

HD 29907

The Ca I abundance is determined from 16 lines with equivalent widths between $18 \mathrm{~m} \AA$ and $174 \mathrm{~m} \AA$. NLTE removes a trend with the line strength displayed by LTE abundances and results in the smaller standard deviation, $\sigma=0.05 \mathrm{dex}$, compared to $\sigma=0.08$ dex in the LTE case. Mean NLTE $\left(S_{\mathrm{H}}=0.1\right)$ and LTE abundances agree within 0.01 dex.

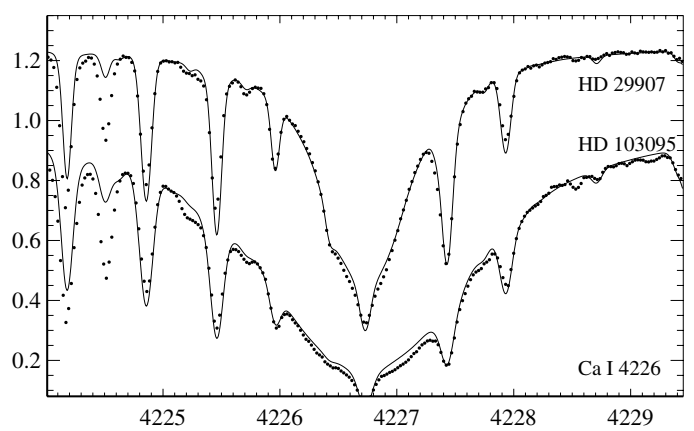

Fig. 9. Theoretical NLTE $\left(S_{\mathrm{H}}=0.1\right.$, continuous line) profiles of the Ca I $\lambda 4226$ line compared with the observed spectra of HD 29907 and HD 103095 (bold dots). For each star, the Ca abundance taken from the analysis of the CaI subordinate lines (see Table 5) fits the resonance line as well. Offsets in y are applied for better illustration.

\section{HD 59392}

The NLTE and LTE mean abundances derived from $17 \mathrm{Ca}$ I lines turn out to be quite similar, $[\mathrm{Ca} / \mathrm{Fe}]\left(S_{\mathrm{H}}=0.1\right)=0.26 \pm 0.04$ and $[\mathrm{Ca} / \mathrm{Fe}](\mathrm{LTE})=0.24 \pm 0.05$.

Our calculations show that, for every star, overionization in the atmospheric layers below $\log \tau_{5000}=-1$ (Fig. 2) leads to weakening the line wings of $\lambda 4226$ compared to the LTE case, while a steep decrease in the departure coefficient ratio $b(4 \mathrm{p}) / b(4 \mathrm{~s})$ above that depth point results in the opposite effect for the line core. In the two coolest stars of our small sample, HD 29907 and HD 103095, the Ca I resonance line is very strong with the core formed in the uppermost atmospheric layers near $\log \tau_{5000}=-4.4$ and -4.9 , respectively (we indicate here a location of line-center optical-depth unity). However, total line absorption is dominated by the van der Waals broadened wings and not by the core. For both stars, we find that the NLTE theoretical profile computed with $\mathrm{Ca}$ abundance determined from the Ca I subordinate lines describes the observed profile well, except for the very core, which can be influenced by the star's chromosphere. Results are illustrated in Fig. 9.

For HD 84937 and HD 19445, the agreement between subordinate lines of $\mathrm{Ca}$ I, Ca II 8498, and Ca I 4226 is excellent when $S_{\mathrm{H}}=0.1$ is assumed. Ca I 4226 requires a $[\mathrm{Ca} / \mathrm{Fe}]$ ratio of 0.41 for HD 84937 and of 0.36 for HD 19445.

A less consistent picture emerges for the remaining three stars. When $\mathrm{Ca}$ abundance is fixed at the value derived from the analysis of the CaI subordinate lines, the half-width of the theoretical NLTE profile of $\lambda 4226$ is found to be larger than the observed one. To fit the observed line width, a smaller Ca abundance is required: by 0.08 dex for HD 59292, 0.09 dex for HD 140283, and 0.22 dex for BD-4 3208 . Discrepancies are smaller in LTE for these stars, but larger for e.g. HD 84937. The found discrepancies could well be related to uncertainties in the microturbulence value, because, in each star investigated, Ca I 4226 lies on the saturated part of the curve of growth, and $\Delta V_{\text {mic }}=-0.1 \mathrm{~km} \mathrm{~s}^{-1}$ translates to $\Delta \log \varepsilon(\lambda 4226)=$ +0.03 dex to +0.04 dex for different stars. However, for a star like BD $-4^{\circ} 3208$, a substantial reduction of the microturbulence would be required, which in turn would affect the good agreement between $\mathrm{Ca}$ I and Ca II reported above. We note in passing that a similar discrepancy is found for subordinate lines of $\mathrm{Mg}$ I and the $\mathrm{Mg} \mathrm{I} b$ triplet lines in these stars.

Are these then shortcomings of our SE calculations? Collisions are inefficient in the statistical equilibrium of atoms in the layers, where the Doppler core of $\lambda 4226$ is formed, between $\log \tau_{5000}=-1$ and -3 , for the most metal-poor stars of our 
sample, BD $-4^{\circ} 3208$ and HD 140283. The related non-LTE effect is therefore entirely due to photoionization, which should be modelled accurately (given the correctness of the OP photoionization cross-sections.) The explanation can lie with the adopted one-dimensional atmospheric models. Based on the recent results of Shchukina et al. (2005) for the weak and moderately strong $\left(W_{\lambda} \leq 80 \mathrm{~m} \AA\right.$ ) lines of Fe I in HD 140283, we expect an overall small effect of atmospheric-temperature and velocity inhomogeneities on the Ca I subordinate lines in our sample of metal-poor stars. The CaI resonance line may be a different case, because it is formed over the more extended range of atmospheric depths. For a fully quantitative understanding of its formation in cool stars, more studies are required on the basis of advanced model atmospheres. One clear advantage of such studies will be the removal of adjustable parameters like microturbulence, from which the current modelling potentially suffers.

\section{The $\mathrm{Ca}$ I/Ca II ionization equilibrium as indicator of surface gravity for extremely metal-poor stars}

In this section, we consider the possibility of using the $W(\mathrm{Ca}$ I 4226)/W(Ca II 3933) and $W(\mathrm{Ca}$ I 4226)/W(Ca II 8498) equivalent width ratios as indicators of surface gravity for extremely metal-poor stars. At extremely low $\mathrm{Ca}$ abundance, the line wings contribute no longer to $W(\mathrm{Ca}$ II 8498$)$. The line is expected to be strengthened with decreasing $\log g$ due to decreasing the $\mathrm{H}^{-}$continuous absorption. The NLTE effects for $\mathrm{Ca}$ II $\lambda 8498$ are amplified in the same direction. Thus, the $W(\mathrm{Ca}$ I 4226$) / W(\mathrm{Ca}$ II 8498$)$ ratio should increase when $\log g$ goes up. The Ca II resonance lines remain strong even at $[\mathrm{Ca} / \mathrm{H}]=-5$, and their van der Waals broadened wings are weakened with decreasing $\log g$. The $\mathrm{Ca}$ I resonance line is weakened in the same direction due to amplified overionization. Thus, the $W(\mathrm{Ca}$ I 4226) / $W(\mathrm{Ca}$ II 3933) ratio is rather insensitive to any variation in $\log g$. Both ratios were calculated for a small grid of models with $[\mathrm{Fe} / \mathrm{H}]=-4.34$; the $\mathrm{Ca}$ abundance was adopted to be $[\mathrm{Ca} / \mathrm{H}]=-4.9$. The results corresponding to $T_{\text {eff }}=5500 \mathrm{~K}$ are plotted in Fig. 10 as a function of $\log g$. It is clear that the $W(\mathrm{Ca}$ I 4226$) / W(\mathrm{Ca}$ II 8498$)$ ratio can be used to determine the surface gravity for extremely metal-poor stars, contrary to the ratio involving the $\mathrm{Ca}$ II resonance line(s), which is nearly constant over the $\log g$ range between 2.5 and 4.5. An application of this technique to the two known ultra-metal-poor stars (HE 0107-5240, Christlieb et al. 2002; HE 1327-2326, Frebel et al. 2005) will be presented in a forthcoming paper.

\section{Concluding remarks}

In this study, NLTE line formation of an extended list of $\mathrm{Ca}$ I and $\mathrm{Ca}$ II lines was considered for the temperatures ranging between $T_{\text {eff }}=5000 \mathrm{~K}$ and $T_{\text {eff }}=6000 \mathrm{~K}$, for surface gravities $\log g=3.0$ and 4.0, and for metallicities from $[\mathrm{Fe} / \mathrm{H}]=0$ down to $[\mathrm{Fe} / \mathrm{H}]=-4.34$. For every $\mathrm{Ca}$ I line, departures from LTE affect its profile significantly over the whole range of stellar parameters. If a line is strong (multiplets $2,3,4,18,21$, and 47 at $[\mathrm{Fe} / \mathrm{H}] \geq-2$ and the remaining multiplets at $[\mathrm{Fe} / \mathrm{H}] \geq-1$ ), its wings are weakened but the core is strengthened compared to the LTE case. The value and sign of the NLTE abundance correction are defined by a relative contribution of the core and the wings to the overall line strength. When the line becomes weak due to decreasing $\mathrm{Ca}$ abundance, NLTE leads to depleted total absorption in the line and positive abundance correction. The NLTE effects are very large at extremely low $\mathrm{Ca}$ abundance.

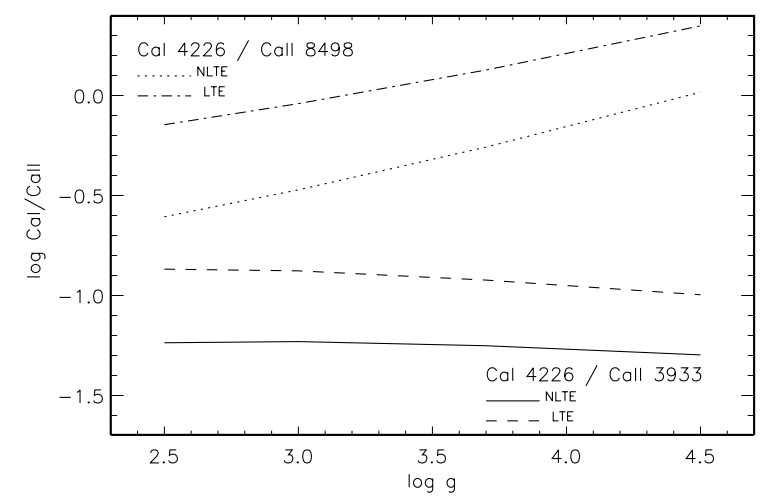

Fig. 10. The Ca I 4226 / Ca II 3933 and Ca I 4226 / Ca II 8498 equivalentwidth ratios as a function of the surface gravity for the models with $T_{\text {eff }}=5500 \mathrm{~K}$ and $[\mathrm{Fe} / \mathrm{H}]=-4.34$. A Ca abundance of $[\mathrm{Ca} / \mathrm{H}]=-4.9$ has been adopted everywhere.

For example, at $[\mathrm{Ca} / \mathrm{H}]=-4.9$ NLTE abundance correction for Ca I $\lambda 4226$ can exceed 0.5 dex. Thus, for a given line, $\Delta_{\text {NLTE }}$ depends on $T_{\text {eff }}, \log g,[\mathrm{Ca} / \mathrm{H}]$, and microturbulence value, and, for a given model, $\Delta_{\text {NLTE }}$ is different in value and sign for a variety of $\mathrm{Ca}$ I lines. Any interpolation of NLTE results for $\mathrm{Ca}$ I has to be performed with caution.

A different situation is found for Ca II. For every Ca II line, NLTE leads to enhanced absorption in the line core and negative abundance correction over the whole range of stellar parameters. The absolute value of $\Delta_{\text {NLTE }}$ is defined by the relative contribution of the core and the wings to equivalent width. For example, NLTE corrections remain very small $(\leq 0.02 \mathrm{dex})$ for the resonance lines and grow in absolute value with decreasing $\mathrm{Ca}$ abundance for the IR lines of multiplet $3 d-4 p$ exceeding 0.4 dex in a metal-poor model with $T_{\text {eff }}=6000 \mathrm{~K}, \log g=3.0$, and $[\mathrm{Fe} / \mathrm{H}]=$ -3 . Such corrections are important to consider for correctly interpreting the near-IR spectroscopic data collected in the RAVE survey and the future ESA Gaia satellite mission.

Empirical evidence is found from the analysis of stellar spectra that inelastic collisions with hydrogen atoms serve as an additional source of thermalization in cool (metal-poor) stars. Our best choice for a scaling factor to the formula of Steenbock \& Holweger (1984) for calculating hydrogenic collisions is $S_{\mathrm{H}}=$ 0.1 . Disregarding hydrogenic collisions completely will lead to an overestimated NLTE effect at low metallicity.

Taking advantage of our SE approach and accurate atomic data for the investigated lines, we obtain good agreement, within 0.04 dex, between absolute solar Ca I (from 23 lines) and Ca II (from 8 lines) abundances, and the average of the two is $\log \varepsilon_{\mathrm{Ca}}^{\odot}=$ $6.38 \pm 0.06$. Likewise, calcium abundances from two ionization stages are examined for the first time for five metal-poor stars. We show that NLTE largely removes obvious discrepancies between $\mathrm{Ca}$ I and Ca II obtained under the LTE assumption.

Solving the restricted NLTE problem for calcium in "classical" one-dimensional LTE model atmospheres, we qualitatively understand the formation of the $\mathrm{Ca} I$ resonance line in metal-poor stars, where it has a purely photospheric origin. In agreement with observations, NLTE predicts weakening of the line wings and strengthening of the line core compared to the LTE case. Residual discrepancies may be related to the use of classical model atmospheres with adjustable parameters like microturbulence.

Acknowledgements. We are grateful to Thomas Gehren for providing a Windows version of the code DETAIL and Tatyana Ryabchikova for help with collecting atomic data. L.M. acknowledges with gratitude the Institute 
of Astronomy and Astrophysics of Munich University for warm hospitality during a productive stay in May-August 2005. This research was supported by the Deutsche Forschungsgemeinschaft with grant 436 RUS 17, the Russian Foundation for Basic Research with grant 05-02-39005-GFEN-a, the Royal Swedish Academy of Sciences with grant 11630102, and the Presidium RAS Programme "Origin and evolution of stars and the Galaxy". A.J.K. acknowledges support from the Leopoldina foundation/Germany under grant BMBF-LPD 9901/8-87 and the Swedish Research Council. A.J.K. also thanks Nikolai Piskunov for travel support for a visit to Moscow in April 2005. We thank the anonymous referee for valuable suggestions and comments. We made ample use of data collected in the NIST and VALD databases.

\section{References}

Allen, C. W. 1973, Astrophysical Quantities, Athlone Press Allende Prieto, C., Asplund, M., Garsia Lopez, R. J., \& Lambert, D. 2002, ApJ, 567,544

Anders, E., \& Grevesse, N. 1989, Geoch. Cosmochim. Acta, 53, 197

Andretta, V. Busa, I., Gomez, M. T., \& Terranegra, L. 2005, A\&A, 430, 669

Anstee, S. D., \& O'Mara, B. J. 1995, MNRAS, 276, 859 (A\&O'M)

Aoki, W., Frebel, A., Christlieb, N., et al. 2006, ApJ, 639, 897

Armandroff, T. E., \& da Costa, G. S. 1991, AJ, 101, 1329

Asplund, M., Grevesse, N., \& Sauval, A. J. 2005, ASP Conf. Ser., 336, 25

Aufdenberg, J. P., Ludwig, H.-G., \& Kervella, P. 2005, ApJ, 633, 424

Bagnulo, S., Jehin, E., Ledoux, C., et al. 2003, ESO Messenger, 114, 10

Barklem, P. S., \& O'Mara, B. J. 1997, MNRAS, 290, $102\left(A \& O^{\prime} M\right)$

Barklem, P. S., \& O'Mara, B. J. 1998, MNRAS, 300, 863 (A\& $\left.O^{\prime} M\right)$

Barklem, P. S., \& Aspelund-Johansson, J. 2005, A\&A, 435, 373

Barklem, P. S., O'Mara, B. J., \& Ross, J. E. 1998, MNRAS, 296, 1057 (A\&O'M)

Belyaev, A. K., Grosser, J., Hahne, J., \& Menzel, T. 1999, Phys. Rev. A, 60, 2151

Belyaev, A. K., \& Barklem, P. 2003, Phys. Rev. A, 68, 062703

Burgess, A., Chidichimo, M. C., \& Tully, J. A. 1995, A\&A, 300, 627

Butler, K., \& Giddings, J. 1985, Newsletter on the analysis of astronomical spectra No. 9, University of London

Castelli, F., Gratton, R. G., \& Kurucz, R. L. 1997, A\&A, 318, 841

Cayrel, R., Depagne, E., Spite, M., et al. 2004, A\&A, 416, 1117

Cenarro, A. J., Cardiel, N., Gorgas, J., et al. 2001, MNRAS, 326, 959

Christlieb, N., Bessel, M., Beers, T., et al. 2002, Nature, 419, 904

Cunto, W., \& Mendoza, C. 1992, Rev. Mex. Astrofis., 23, 107

Doyle, R. O. 1968, ApJ, 153, 987

Drake, J. 1991, MNRAS, 251, 369

Drawin, H. W. 1968, Z. Phys., 211, 404

Drawin, H. W. 1969, Z. Phys., 225, 483

Drozdowski, R., Ignaciuk, M., Kwela, J., \& Heldt, J. 1997, Z. Phys. D, 41, 125

Frebel, A., Aoki, W., Christlieb, N., et al. 2005, Nature, 434, 871

Fuhrmann, K. 1998, A\&A, 338, 161

Fuhrmann, K., Pfeiffer, M., Frank, C., Reetz, J., \& Gehren, T. 1997, A\&A, 323, 909

Gehren, T. 1975, A\&A, 38, 289

Gehren, T., Liang, Y. C., Shi, J. R., Zhang, H. W., \& Zhao, G. 2004, A\&A, 413, 1045
Ibata, R., Chapman, S., Ferguson, A. M. N., et al. 2005, ApJ, 634, 287

Idiart, T. P., \& Thevenin, F. 2000, ApJ, 541, 207

Jørgensen, U. G., Carlsson, M., \& Johnson, R. 1992, A\&A, 254, 258

Kerkeni, B., Barklem, P. S., Spielfiedel, A., \& Feautrier, N. 2004, J. Phys. B, 37 , 677

Korn, A., Shi, J., \& Gehren, T. 2003, A\&A, 407, 691

Kupka, F., Piskunov, N., Ryabchikova, T. A., Stempels, H. C., \& Weiss, W. W. 1999, A\&AS, 138, 119

Kurucz, R. L. 1992, CD-ROM No. 18; CD-ROM No. 23

Kurucz, R. L., Furenlid, I., Brault, J., \& Testerman, L. 1984, Solar Flux Atlas from 296 to $1300 \mathrm{~nm}$. Nat. Solar Obs., Sunspot, New Mexico

Lucas, D. M., Ramos, A., Home, J. P., et al. 2004, Phys. Rev. A, 69, 012711

Mallik, S. V. 1997, A\&AS, 124, 359

Mashonkina, L. I. 1996, Model Atmospheres and Spectrum Synthesis, ASP Conf. Ser., 108, 140

Mashonkina, L., Gehren, T., Travaglio, C., \& Borkova, T. 2003, A\&A, 397, 275

Moore, C. E. 1972, A Multiplet Table of Astrophysical Interest, NSRDS-NBS 40

Nissen, P. E., Primas, F., Asplund, M., \& Lambert, D. L. 2002, A\&A, 390, 235

Nörtershäuser, W., Blaum, K., Icker, K., et al. 1998, Eur. Phys. J. D, 2, 33

Perryman, M. A. C., de Boer, K. S., Gilmore, G., et al. 2001, A\&A, 369, 339

Ramirez, I., \& Melendez, J. 2005, ApJ, 626, 446

Rutledge, G. A., Hesser, J. E., \& Stetson, P. B. 1997, PASP, 109, 907

Rybicki, G. B., \& Hummer, D. G. 1991, A\&A, 245, 171

Rybicki, G. B., \& Hummer, D. G. 1992, A\&A, 262, 209

Saglia, R. P., Maraston, C., Thomas, D., Bender, R., \& Colless, M. 2002, ApJ, 579, L13

Samson, A. M., \& Berrington, K. A. 2001, ADNDT, 77, 87

Seaton, M. J. 1962, Proc. Phys. Soc. London, 79, 1105

Seaton, M. J. 1962, in Atomic and Molecular Processes (New York: Academic Press)

Seaton, M. J., Mihalas, D., \& Pradhan, A. K. 1994, MNRAS, 266, 805

Shabanova, L. N. 1963, Opt. Spectros. (USSR), 15, 450

Shchukina, N. G., Trujillo Bueno, J., \& Asplund, M. 2005, ApJ, 618, 939

Shine, R. A., \& Linsky, J. L. 1974, Solar Phys., 39, 49

Smith, G. 1981, A\&A, 103, 351

Smith, G. 1988, J. Phys. B, 21, 2827

Smith, W. W., \& Gallagher, A. 1966, Phys. Rev., 145, 26

Smith, G., \& O’Neil, J. A. 1975, A\&A, 38, 1

Smith, G., \& Raggett, D. St. J. 1981, J. Phys. B, 14, 4015

Spielfiedel, A., Feautrier, N., Chambaud, G., \& Levy, B. 1991, J. Phys. B, 24, 4711

Steenbock, W., \& Holweger, H. 1984, A\&A, 130, 319

Steinmetz, M., Zwitter, T., Siebert, A., et al. 2006, AJ, 132, 1645

Sugar, J., \& Corliss, C. 1985, J. Phys. Chem. Ref. Data, 14, Suppl. No. 2

Theodosiou, C. E. 1989, Phys. Rev. A, 39, 4880

Tolstoy, E., Irwin, M. J., Cole, A. A., et al. 2001, MNRAS, 327, 918

VandenBerg, D. A., Swenson, F. J., Rogers, F. J., Iglesias, C. A., \& Alexander, D. R. 2000, ApJ, 532, 430

van Regemorter, H. 1962, ApJ, 136, 906

Watanabe, T., \& Steenbock, W. 1985, A\&A, 149, 21 